\title{
Tracing the cold and warm physico-chemical structure of deeply embedded protostars: IRAS 16293-2422 vs. VLA 1623-2417
}

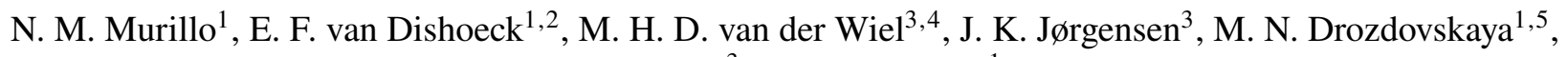 \\ H. Calcutt ${ }^{3}$, and D. Harsono ${ }^{1}$ \\ ${ }^{1}$ Leiden Observatory, Leiden University, PO Box 9513, 2300 RA Leiden, The Netherlands \\ e-mail: nmurillo@strw.leidenuniv.nl \\ 2 Max-Planck-Institut für extraterrestrische Physik, Giessenbachstraße 1, 85748 Garching bei München, Germany \\ ${ }^{3}$ Centre for Star and Planet Formation, Niels Bohr Institute \& Natural History Museum of Denmark, University of Copenhagen, \\ Øster Voldgade 5-7, 1350 Copenhagen K., Denmark \\ 4 ASTRON, the Netherlands Institute for Radio Astronomy, Postbus 2, 7990 AA Dwingeloo, The Netherlands \\ ${ }^{5}$ Center for Space and Habitability (CSH), University of Bern, Sidlerstrasse 5, 3012 Bern, Switzerland
}

Received 5 August 2017 / Accepted 29 April 2018

\begin{abstract}
Context. Much attention has been placed on the dust distribution in protostellar envelopes, but there are still many unanswered questions regarding the physico-chemical structure of the gas.

Aims. Our aim is to start identifying the factors that determine the chemical structure of protostellar regions, by studying and comparing low-mass embedded systems in key molecular tracers.

Methods. The cold and warm chemical structures of two embedded Class 0 systems, IRAS 16293-2422 and VLA $1623-2417$ were characterized through interferometric observations. $\mathrm{DCO}^{+}, \mathrm{N}_{2} \mathrm{H}^{+}$, and $\mathrm{N}_{2} \mathrm{D}^{+}$were used to trace the spatial distribution and physics of the cold regions of the envelope, while $\mathrm{c}-\mathrm{C}_{3} \mathrm{H}_{2}$ and $\mathrm{C}_{2} \mathrm{H}$ from models of the chemistry are expected to trace the warm (UV-irradiated) regions.

Results. The two sources show a number of striking similarities and differences. $\mathrm{DCO}^{+}$consistently traces the cold material at the disk-envelope interface, where gas and dust temperatures are lowered due to disk shadowing. $\mathrm{N}_{2} \mathrm{H}^{+}$and $\mathrm{N}_{2} \mathrm{D}^{+}$, also tracing cold gas, show low abundances toward VLA 1623-2417, but for IRAS 16293-2422, the distribution of $\mathrm{N}_{2} \mathrm{D}^{+}$is consistent with the same chemical models that reproduce $\mathrm{DCO}^{+}$. The two systems show different spatial distributions $\mathrm{c}^{-} \mathrm{C}_{3} \mathrm{H}_{2}$ and $\mathrm{C}_{2} \mathrm{H}$. For IRAS 16293-2422, c $-\mathrm{C}_{3} \mathrm{H}_{2}$ traces the outflow cavity wall, while $\mathrm{C}_{2} \mathrm{H}$ is found in the envelope material but not the outflow cavity wall. In contrast, toward VLA 1623-2417 both molecules trace the outflow cavity wall. Finally, hot core molecules are abundantly observed toward IRAS 16293-2422 but not toward VLA 1623-2417.

Conclusions. We identify temperature as one of the key factors in determining the chemical structure of protostars as seen in gaseous molecules. More luminous protostars, such as IRAS 16293-2422, will have chemical complexity out to larger distances than colder protostars, such as VLA 1623-2417. Additionally, disks in the embedded phase have a crucial role in controlling both the gas and dust temperature of the envelope, and consequently the chemical structure.
\end{abstract}

Key words. astrochemistry - stars: formation - stars: low-mass - stars: individual: IRAS 16293-2422 - methods: observational stars: individual: VLA 1623-2417

\section{Introduction}

While there is a well-established outline of the physical evolution of protostellar systems (Evans 1999; Dunham et al. 2014; Li et al. 2014; Reipurth et al. 2014), there are still many questions regarding the physico-chemical structure of these systems. Several studies point out the chemical richness and diversity of young embedded protostars, most notably in the Class 0 stage, ranging from simple molecules to carbon chains and complex organics (see reviews by Herbst \& van Dishoeck 2009; Caselli \& Ceccarelli 2012; Sakai \& Yamamoto 2013). In contrast, some other protostellar systems show much less chemical complexity (e.g., Jørgensen et al. 2005b; Maret et al. 2006; Öberg et al. 2014; Lindberg et al. 2014b, 2016, 2017; Fayolle et al. 2015; Bergner et al. 2017), a situation made more extreme when some starless cores have stronger molecular line emissions than the already formed protostars (e.g., Bergman et al. 2011; Bacmann et al. 2012; Friesen et al. 2014). It is interesting to explore the chemical structure and evolution of early stage protostars and what physical quantities dictate the resulting chemical structure as observed in the gas phase.

The chemical fingerprint generated in the early embedded stages of star formation may be transmitted to the later stages and eventually the protoplanetary disk, where planets and comets are formed (e.g., Aikawa et al. 1999; Aikawa \& Herbst 1999; Visser et al. 2009, 2011; Hincelin et al. 2013; Drozdovskaya et al. 2014; Willacy et al. 2015; Yoneda et al. 2016). Which factors then generate a protostellar system's fingerprint? Protostellar cores may inherit their chemical composition from the parent clouds that eventually collapse to form protostars (e.g., Visser et al. 2009, 2011; Aikawa et al. 2012; Furuya et al. 2012; Tassis et al. 2012; Hincelin et al. 2016). It would then seem likely that protostars from the same parent cloud would have a similar chemical composition. However, this would require the cloud to have a homogeneous composition, which is not always the case (Bergman et al. 2011). Instead, other mechanisms 
could alter the chemical fingerprint. Turbulence and large-scale motions could stir the gas and dust of the cloud core around, moving material from the outer region of the core closer to the warmer regions of the system, kick-starting chemical reactions and producing enhancements of selected species. Formation of more complex chemical species is likely to occur through grain-surface reactions (i.e., on ice and dust surfaces) instead of in the gas-phase, and such reactions proceed faster at higher dust temperatures which increases the mobility of radicals (Garrod \& Herbst 2006). If material near the outflow cavities is warmer than elsewhere in the envelope, this could generate pockets of chemically rich ices that, once heated above the sublimation temperature, would be released into the gas-phase (Drozdovskaya et al. 2015). Moreover, UV radiation can photodissociate CO and create free atomic carbon that leads to efficient formation of carbon-bearing molecules. UV irradiation, together with age and variations in accretion rates, would also produce different outcomes, even with the same initial ingredients. In addition, simple warm-chemistry molecules can be the precursors to more complex molecules (Sakai \& Yamamoto 2013).

The physical evolution of the individual protostars, for example, the collapse time and structure, will also impact the chemical fingerprint. An important consideration regarding the physical structure is that disks may have formed already in the early stages, as shown by recent observations (e.g., Tobin et al. 2012; Murillo et al. 2013; Harsono et al. 2014; Codella et al. 2014; Lindberg et al. 2014a; Yen et al. 2017). Not only do disks provide a high density long-lived reservoir preventing molecules from falling into the star, but they also affect the thermal structure of their surroundings. Thus, the disk-envelope interface and the envelope itself must be studied (Murillo et al. 2015; Persson et al. 2016). The disk-envelope interface and the outer envelope of embedded systems are traced by cold-chemistry molecules, since these regions are usually shielded from heating by the central protostar (van Dishoeck et al. 1995; Jørgensen et al. 2004, 2005a; Sakai et al. 2014b; Murillo et al. 2015). Through the study of molecules sensitive to temperature, we can then understand the structure of embedded protostellar systems.

Aiming to explore the chemical evolution of the earliest embedded protostellar systems - Class 0 - we compare two systems from $\rho$ Ophiuchus $(d \sim 120$ pc; Loinard et al. 2008), IRAS 16293-2422 and VLA 1623-2417 (Fig. 1), separated by a projected distance of $2.8 \mathrm{pc}$. Recent measurements to these two systems place IRAS 16293-2422 at a distance of $141 \pm$ $30 \mathrm{pc}$ (Dzib et al. 2018) and VLA 1623-2417 at $137.3 \pm$ 1.2 pc (Ortiz-León et al. 2017). However, for ease of comparison with previous work, we adopt the distance of $120 \mathrm{pc}$ for both sources. Most previous studies of IRAS 16293-2422 and VLA 1623-2417 were based on single-dish observations. The advent of the Atacama Large Millimeter/submillimeter Array (ALMA) now allows chemical studies on 100 AU scales that spatially resolve the different physical components of the system.

IRAS 16293-2422 (hereafter IRAS 16293) is a widely studied multiple system, located in $\mathrm{L} 1689 \mathrm{~N}$, with a complicated outflow structure being driven by source A (Stark et al. 2004; Yeh et al. 2008; Loinard et al. 2013; Kristensen et al. 2013; Girart et al. 2014). IRAS 16293 A and B, separated by about 620 AU (Fig. 1), have different inclination angles, with the disk-like structure of A being inclined, and $\mathrm{B}$ being oriented face-on with respect to the line of sight (Pineda et al. 2012; Jørgensen et al. 2016). Due to the different inclination angles, it is difficult to determine whether these systems are at the same evolutionary stage or not (Murillo et al. 2016). Both components are chemically rich but show differences in structure (Bottinelli et al. 2004; Bisschop et al. 2008; Jørgensen et al. 2011).

VLA 1623-2417 (hereafter VLA 1623) is a triple protostellar system, located in L1688 ( $\rho$ Oph A), mostly studied for its prominent outflow in the region (André et al. 1990; Caratti o Garatti et al. 2006). The three components of the system, VLA 1623 A, B, and W are separated by 132 and 1200 AU (Fig. 1), respectively, have similar inclination angles, and have also been found to be at different evolutionary stages (Murillo \& Lai 2013; Murillo et al. 2013). VLA 1623 has been shown to be largely line poor in single-dish studies (Garay et al. 2002; Jørgensen et al. 2004; Bergman et al. 2011; Friesen et al. 2014).

In this paper, we present observations of $\mathrm{DCO}^{+}, \mathrm{N}_{2} \mathrm{H}^{+}$, $\mathrm{N}_{2} \mathrm{D}^{+}, \mathrm{c}-\mathrm{C}_{3} \mathrm{H}_{2}$, and $\mathrm{C}_{2} \mathrm{H}$ toward IRAS 16293 and VLA 1623, using ALMA, the Submillimeter Array (SMA; Ho et al. 2004), and the Atacama Pathfinder EXperiment (APEX; Güsten et al. 2006). $\mathrm{DCO}^{+}, \mathrm{N}_{2} \mathrm{H}^{+}$, and $\mathrm{N}_{2} \mathrm{D}^{+}$are known to be good tracers of cold gas where $\mathrm{CO}$ is frozen out. $\mathrm{c}-\mathrm{C}_{3} \mathrm{H}_{2}$ and $\mathrm{C}_{2} \mathrm{H}$ are usually seen in photon-dominated regions (PDRs) such as the Orion Bar (Pety et al. 2007; van der Wiel et al. 2009; Nagy et al. 2015) and the Horsehead Nebula (Cuadrado et al. 2015; Guzmán et al. 2015), with both species located at the irradiated, and thus warmer, edge of these regions. $\mathrm{c}-\mathrm{C}_{3} \mathrm{H}_{2}$ and $\mathrm{C}_{2} \mathrm{H}$ could thus be expected to trace the (UV-irradiated) outflow cavity walls, although both species have also been found just outside the disk-envelope interface (Sakai et al. 2014a). Besides mapping their distributions, multiple lines from a single molecule can also be used to trace physical conditions such as temperature and density (van Dishoeck et al. 1993; Evans 1999; van der Tak et al. 2007; Shirley 2015) and the current dataset allows this to be done for several species.

Details of the observations with ALMA, SMA, and APEX are described in Sect. 2. Section 3 describes the spatial distribution of each molecule for both systems. The observations are compared to chemical models and physical parameters are derived in Sect. 4. Sections 5 and 6 compare both systems studied here with other objects found in literature and place the results of our work in context.

\section{Observations}

\subsection{IRAS 16293-2422}

IRAS 16293 was targeted in the Protostellar Interferometric Line Survey (PILS) program (Project-ID: 2013.1.00278.S; PI: Jes K. Jørgensen; Jørgensen et al. 2016), an ALMA Cycle 2 unbiased spectral survey in Band 7, using both the $12 \mathrm{~m}$ array and the Atacama Compact Array (ACA). The spectral setup covers a frequency range from 329.147 to $362.896 \mathrm{GHz}$, and provides a velocity resolution of $0.2 \mathrm{~km} \mathrm{~s}^{-1}$. The phase center was $\alpha_{J 2000}=16: 32: 22.72 ; \delta_{J 2000}=-24: 28: 34.3$, set to be equidistant from the two sources A and B at $v_{\mathrm{lsr}}=3.1$ and $2.7 \mathrm{~km} \mathrm{~s}^{-1}$ (Jørgensen et al. 2011), respectively. The resulting $(u, v)$ coverage of the combined $12 \mathrm{~m}$ array and the ACA observations are sensitive to the distribution of material with an extent of up to $13^{\prime \prime}$ and a circular synthesized beam of $0.5^{\prime \prime}$. A detailed description of the observations and reduction is given in Jørgensen et al. (2016). Many complex molecules detected in this survey (e.g., Coutens et al. 2016; Jørgensen et al. 2016; Lykke et al. 2017) show relatively compact emission peaking close to the location of the two protostars. This work focuses on a few of the molecules detected in the spectral survey that show extended emission, namely, $\mathrm{DCO}^{+}, \mathrm{c}-\mathrm{C}_{3} \mathrm{H}_{2}$, and $\mathrm{C}_{2} \mathrm{H}$. Several transitions of $\mathrm{c}-\mathrm{C}_{3} \mathrm{H}_{2}$ are present throughout the frequency range of PILS, 
Table 1. Summary of line observations.

\begin{tabular}{|c|c|c|c|c|c|c|c|c|}
\hline \multirow[b]{2}{*}{ Line } & \multirow[b]{2}{*}{ Transition } & \multirow[b]{2}{*}{$\begin{array}{c}v \\
(\mathrm{GHz})\end{array}$} & \multirow[b]{2}{*}{$\log _{10} A_{i j}$} & \multirow[b]{2}{*}{$\begin{array}{l}E_{\text {up }} \\
(\mathrm{K})\end{array}$} & \multicolumn{2}{|c|}{ IRAS 16293-2422 } & \multicolumn{2}{|c|}{ VLA 1623-2417 } \\
\hline & & & & & 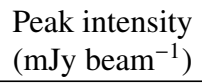 & $\begin{array}{c}\text { Line width } \\
\left(\mathrm{km} \mathrm{s}^{-1}\right)\end{array}$ & 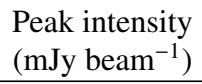 & $\begin{array}{c}\text { Line width } \\
\left(\mathrm{km} \mathrm{s}^{-1}\right)\end{array}$ \\
\hline \multicolumn{9}{|c|}{ ALMA } \\
\hline $\mathrm{c}-\mathrm{C}_{3} \mathrm{H}_{2}$ & $6_{0,6}-5_{1,5}$ & 217.822148 & -3.23 & 38.61 & & $\ldots$ & 194 & 0.5 \\
\hline $\mathrm{c}-\mathrm{C}_{3} \mathrm{H}_{2}^{2}$ & $5_{5,0}-4_{4,1}$ & 349.26400 & -2.78 & 48.98 & $240^{a}$ & 0.5 & $\ldots$ & $\ldots$ \\
\hline $\mathrm{c}-\mathrm{C}_{3} \mathrm{H}_{2}$ & $10-9^{b}$ & 351.78158 & -2.61 & 96.49 & $410^{a}$ & 0.6 & $\ldots$ & $\ldots$ \\
\hline $\mathrm{c}-\mathrm{C}_{3}^{3} \mathrm{H}_{2}^{2}$ & $9-8^{c}$ & 351.96597 & -2.67 & 93.34 & $350^{a}$ & 0.6 & $\ldots$ & $\ldots$ \\
\hline $\mathrm{c}-\mathrm{C}_{3} \mathrm{H}_{2}^{2}$ & $8_{2,6}-7_{3,5}$ & 352.18554 & -2.76 & 86.93 & $90^{a}$ & 0.6 & $\ldots$ & $\ldots$ \\
\hline $\mathrm{c}-\mathrm{C}_{3} \mathrm{H}_{2}$ & $8_{3,6}-7_{2,5}$ & 352.19364 & -2.76 & 86.93 & $200^{a}$ & 0.6 & $\ldots$ & $\ldots$ \\
\hline $\mathrm{C}_{2} \mathrm{H}^{2}$ & $4-3 J=9 / 2-7 / 2 \quad F=5-4$ & 349.33771 & -3.88 & 41.91 & 170 & 0.6 & $\ldots$ & $\ldots$ \\
\hline $\mathrm{C}_{2}^{2} \mathrm{H}$ & $4-3 J=9 / 2-7 / 2 F=4-3$ & 349.33899 & -3.89 & 41.91 & 140 & 0.6 & $\ldots$ & $\ldots$ \\
\hline $\mathrm{C}_{2}^{2} \mathrm{H}$ & $4-3 J=7 / 2-5 / 2 F=4-3$ & 349.39927 & -3.90 & 41.93 & 140 & 0.6 & $\ldots$ & $\ldots$ \\
\hline $\mathrm{C}_{2}^{2} \mathrm{H}$ & $4-3 J=7 / 2-5 / 2 F=3-2$ & 349.40067 & -3.92 & 41.93 & 110 & 0.6 & . & $\ldots$ \\
\hline $\mathrm{DCO}^{+}$ & $3-2$ & 216.11258 & -2.62 & 20.74 & $\ldots$ & $\ldots$ & 90 & 0.7 \\
\hline $\mathrm{DCO}^{+}$ & $5-4$ & 360.16978 & -2.42 & 51.86 & 10 & 1.0 & 290 & 0.7 \\
\hline $\mathrm{N}_{2} \mathrm{D}^{+}$ & $3-2$ & 231.32166 & -2.66 & 22.20 & $\ldots$ & $\ldots$ & $<8.58^{d}$ & $\ldots$ \\
\hline $\mathrm{N}_{2}^{2} \mathrm{H}^{+}$ & $4-3$ & 372.67251 & -2.51 & 44.71 & $\ldots$ & $\ldots$ & $<94.9^{d}$ & $\ldots$ \\
\hline \multicolumn{9}{|c|}{ SMA } \\
\hline $\mathrm{DCO}^{+}$ & $3-2$ & 216.11258 & -2.62 & 20.74 & 1800 & 1.0 & $\ldots$ & $\ldots$ \\
\hline $\mathrm{N}_{2} \mathrm{D}^{+}$ & $3-2$ & 231.32166 & -2.66 & 22.20 & 1700 & 2.0 & $\ldots$ & $\ldots$ \\
\hline \multicolumn{9}{|c|}{$\operatorname{APEX}\left(T_{\mathrm{mb}}\right)$} \\
\hline $\mathrm{C}_{2} \mathrm{H}$ & $4-3 J=9 / 2-7 / 2 F=5-4$ & 349.33771 & -3.88 & 41.91 & $\ldots$ & $\ldots$ & $0.96 \mathrm{~K}$ & 0.7 \\
\hline $\mathrm{C}_{2} \mathrm{H}$ & $4-3 J=9 / 2-7 / 2 F=4-3$ & 349.33899 & -3.89 & 41.91 & $\ldots$ & $\ldots$ & $0.62 \mathrm{~K}$ & 0.7 \\
\hline $\mathrm{C}_{2}^{2} \mathrm{H}$ & $4-3 J=7 / 2-5 / 2 F=4-3$ & 349.39927 & -3.90 & 41.93 & $\ldots$ & $\ldots$ & $0.68 \mathrm{~K}$ & 0.7 \\
\hline $\mathrm{C}_{2}^{2} \mathrm{H}$ & $4-3 J=7 / 2-5 / 2 F=3-2$ & 349.40067 & -3.92 & 41.93 & $\ldots$ & $\ldots$ & $0.34 \mathrm{~K}$ & 0.7 \\
\hline $\mathrm{DCO}^{+}$ & $3-2$ & 216.11258 & -2.62 & 20.74 & $\ldots$ & $\ldots$ & $4.8 \mathrm{~K}$ & 0.8 \\
\hline $\mathrm{DCO}^{+}$ & $5-4$ & 360.16978 & -2.42 & 51.86 & $\ldots$ & $\ldots$ & $2.2 \mathrm{~K}$ & 0.8 \\
\hline
\end{tabular}

Notes. ${ }^{(a)} \mathrm{c}-\mathrm{C}_{3} \mathrm{H}_{2}$ peak intensities and line widths taken from the south peak where there is no line confusion. ${ }^{(b)} \mathrm{Blended} 10_{0,10}-9_{1,9}$ and $10_{1,10}-9_{0,9}$ transitions of $\mathrm{c}-\mathrm{C}_{3} \mathrm{H}_{2} \cdot{ }^{(c)}$ Blended $9_{1,8}-8_{2,7}$ and $9_{2,8}-8_{1,7}$ transitions of $\mathrm{c}-\mathrm{C}_{3} \mathrm{H}_{2} \cdot{ }^{(d)} 1 \sigma$ noise level of $\mathrm{N}_{2} \mathrm{D}^{+}$and $\mathrm{N}_{2} \mathrm{H}^{+}$in $0.02 \mathrm{~km} \mathrm{~s}^{-1}$ channel.

References. All rest frequencies were taken from the Cologne Database for Molecular Spectroscopy $\left(\mathrm{CDMS}\right.$; Endres et al. 2016). The c- $\mathrm{C}_{3} \mathrm{H}_{2}$ entry was based on Bogey et al. (1987) with transition frequencies important for our survey from Bogey et al. (1986) and from Spezzano et al. (2012). The CCH entry is based on Padovani et al. (2009) with additional important data from Müller et al. (2000) and Sastry et al. (1981). The $\mathrm{DCO}^{+}$and $\mathrm{N}_{2} \mathrm{H}^{+}$entries are based on Caselli \& Dore (2005) and on Cazzoli et al. (2012), respectively. Information on the $\mathrm{N}_{2} \mathrm{D}^{+}$rest frequency was taken from Pagani et al. (2009a).

however for this work, only five transitions with strong observed emission were chosen in the $349-352 \mathrm{GHz}$ frequency range. For all molecules, the combined $12 \mathrm{~m}$ array and ACA data cubes are used. The typical RMS noise is about 7-10 mJy beam ${ }^{-1}$ per $0.2 \mathrm{~km} \mathrm{~s}^{-1}$ channel, and the flux calibration uncertainty is $\sim 5 \%$ (Jørgensen et al. 2016). Transitions and line frequencies of the molecular species used in this work are listed in Table 1, as well as the peak intensities and line widths. In Table A.1 we list further details of the observations, such as UV-baseline range and largest angular scale recovered in the observations.

Since the PILS survey did not cover lines of $\mathrm{N}_{2} \mathrm{D}^{+} 3-2$ or $\mathrm{DCO}^{+} 3-2$, we include here the observations of these two molecules from a spectral line survey with the SMA (Jørgensen et al. 2011). The phase center was $\alpha_{J 2000}=16: 32: 22.91$; $\delta_{J 2000}=-24: 28: 35.5$. For $\mathrm{DCO}^{+} 3-2$, the beam size is $5.5^{\prime \prime} \times 3.2^{\prime \prime}\left(\mathrm{PA}=17.7^{\circ}\right)$, while for $\mathrm{N}_{2} \mathrm{D}^{+} 3-2$ the beam size is $4.0^{\prime \prime} \times 2.4^{\prime \prime}\left(\mathrm{PA}=-1.0^{\circ}\right)$. The RMS noise is $0.24 \mathrm{Jy} \mathrm{beam}^{-1}$ for a $0.56 \mathrm{~km} \mathrm{~s}^{-1}$ channel width for $\mathrm{DCO}^{+}$, and $0.06 \mathrm{Jy} \mathrm{beam}^{-1}$ for a channel width of $1.1 \mathrm{~km} \mathrm{~s}^{-1}$ for $\mathrm{N}_{2} \mathrm{D}^{+}$. Further details on the reduction and analysis are given in Jørgensen et al. (2011). These data are considered in order to directly compare the cold chemistry of IRAS 16293 with that of VLA 1623. Additionally, two transitions of $\mathrm{DCO}^{+}$are needed to derive temperature and density from line ratios.

\subsection{VLA 1623-2417}

VLA 1623 was observed with ALMA in Cycle 0 using Band 6, with phase center $\alpha_{J 2000}=16: 26: 26.419 ; \delta_{J 2000}=-24: 24: 29.988$. The spectral set-up was configured to observe $\mathrm{DCO}^{+} 3-2$ and $\mathrm{N}_{2} \mathrm{D}^{+} 3-2$ together with $\mathrm{C}^{18} \mathrm{O} 2-1$ and ${ }^{12} \mathrm{CO} 2-1$, providing a velocity resolution of $0.0847 \mathrm{~km} \mathrm{~s}^{-1}$ and a synthesized beam size of $0.85^{\prime \prime} \times 0.56^{\prime \prime}\left(\mathrm{PA}=-83.8^{\circ}\right) . \mathrm{DCO}^{+} 3-2$ data from the Cycle 0 observations were previously presented in Murillo et al. (2015) and are added to this work for completeness in the comparison of both systems. The data reduction results of $\mathrm{C}^{18} \mathrm{O}$ and ${ }^{12} \mathrm{CO}$ can be found in Murillo et al. (2013) and Santangelo et al. (2015).

ALMA Cycle 2 observations of VLA 1623 were carried out in Bands 6 and 7, with phase center $\alpha_{J 2000}=16: 26: 26.390$; $\delta_{J 2000}=-24: 24: 30.688$. Baseline and frequency ranges are listed in Table A.1.

The Cycle 2 Band 6 spectral setup covered $\mathrm{DCO}^{+} 3-2$, $\mathrm{C}^{18} \mathrm{O} 3-2,{ }^{13} \mathrm{CO} 3-2$, and $\mathrm{c}-\mathrm{C}_{3} \mathrm{H}_{2} \quad 6,6-5_{1,5}$ together with continuum. Data calibration was done with J1733-1304 and J1517-2422 for bandpass, J1625-2527 for gain calibration, and J1517-243, J1733-130, Ceres, Mars, and Titan were observed for flux calibration. The spectral windows with line emission have bandwidths of $62 \mathrm{MHz}$ each, while for continuum the total bandwidth is of $8 \mathrm{GHz}$. These observations were carried out 

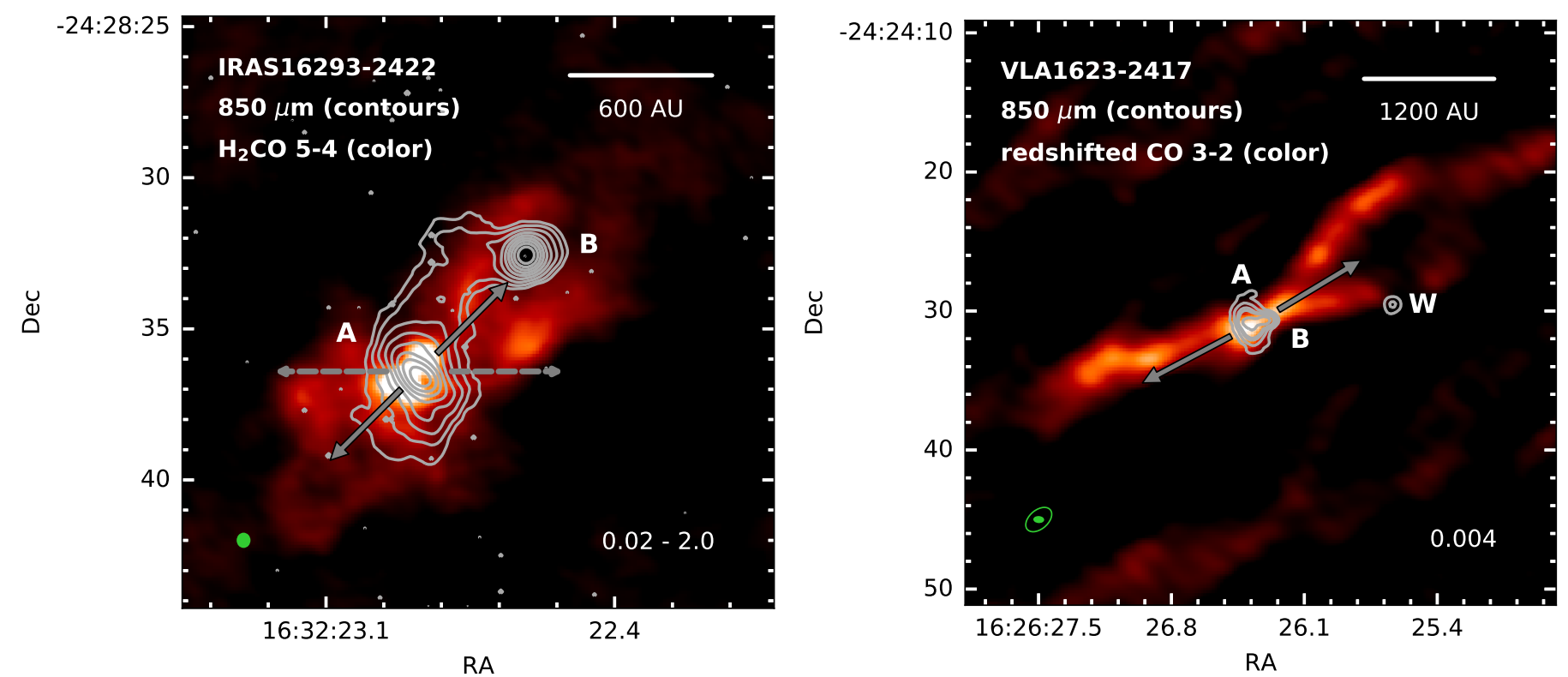

Fig. 1. Continuum at $850 \mu \mathrm{m}$ (contours) for both systems; IRAS 16293-2422 (left panel) and VLA 1623-2417 (right panel), overlaid on intensity integrated $\mathrm{H}_{2} \mathrm{CO}\left(0-60 \mathrm{~km} \mathrm{~s}^{-1}\right.$; color scale $)$ and redshifted $\mathrm{CO}\left(4-15 \mathrm{~km} \mathrm{~s}^{-1}\right.$; color scale), respectively. The green ellipses on the bottom left panel indicate the beam of the observations. For the right panel, the empty ellipse is the beam of the CO observations. For VLA 1623-2417, contours are in steps of $3,8,15,20$, and $50 \sigma$, with $\sigma=0.004 \mathrm{Jy} \mathrm{beam}^{-1}$. For IRAS $16293-2422$, the levels are logarithmically spaced between 0.02 and $2 \mathrm{Jy} \mathrm{beam}^{-1}$, and highlight the ridge that spans between sources A and B. The arrows indicate the direction of red- and blue-shifted outflows from source A in each system.

with both the Total power, ACA, and $12 \mathrm{~m}$ arrays, for a total of four configurations. Two configurations of the $12 \mathrm{~m}$ array, C35-5 $(25 \sim 1000 \mathrm{~m})$ and $\mathrm{C} 34-1(10 \sim 350 \mathrm{~m})$, were used to bridge the gap between the $12 \mathrm{~m}$ and ACA array observations. Cycle 2 Band 6 ACA observations of $\mathrm{DCO}^{+}$were presented in Murillo et al. (2015) and are only included here for visual comparison. The $12 \mathrm{~m}$ and $\mathrm{ACA} \mathrm{C}^{18} \mathrm{O}$ and ${ }^{13} \mathrm{CO}$ observations from Cycle 2 Band 6 will be treated in a future publication. In this work, we focus only on the $12 \mathrm{~m}$ array $\mathrm{c}-\mathrm{C}_{3} \mathrm{H}_{2} 6_{0,6}-5_{1,5}$ observations from Cycle 2 Band 6 data. The C35-5 configuration provides an angular and velocity resolution of $0.45^{\prime \prime} \times 0.25^{\prime \prime}$ $\left(\mathrm{PA}=86.3^{\circ}\right)$ and $0.0208 \mathrm{~km} \mathrm{~s}^{-1}$, respectively, with a typical RMS noise of $7 \mathrm{mJy}^{\text {beam }}{ }^{-1}$. The $\mathrm{C} 34-1$ configuration results in an angular resolution of $1.60^{\prime \prime} \times 0.88^{\prime \prime}\left(\mathrm{PA}=83.8^{\circ}\right)$ with a channel width of $0.0208 \mathrm{~km} \mathrm{~s}^{-1}$ and a typical RMS noise of $20 \mathrm{mJy}$ beam $^{-1}$.

Band 7 observations, with a spectral set-up covering $\mathrm{N}_{2} \mathrm{H}^{+} 5-4, \mathrm{DCO}^{+} 5-4$, and $\mathrm{H}_{2} \mathrm{D}^{+} 1_{1,0}-1_{1,1}$ as well as continuum with only the $12 \mathrm{~m}$ array, provided a spectral and angular resolution of $0.025 \mathrm{~km} \mathrm{~s}^{-1}$ and $0.88^{\prime \prime} \times 0.56^{\prime \prime}\left(\mathrm{PA}=-86.6^{\circ}\right)$, respectively. The spectral windows with line emission have a total bandwidth of $62 \mathrm{MHz}$ each, and continuum has a total bandwidth of $4 \mathrm{GHz}$. Total observing time was $0.9 \mathrm{~h}$ with a $46 \%$ duty cycle, using 34 antennas and a maximum baseline of $350 \mathrm{~m}$. Data calibration was done with J1517-2422, J1625-2527, and Titan for bandpass, gain and flux calibration, respectively. $\mathrm{DCO}^{+}$was detected with a noise of $26 \mathrm{mJy}^{\text {beam }}{ }^{-1}$ per $0.025 \mathrm{~km} \mathrm{~s}^{-1}$. The system temperature was relatively high for the spectral windows containing $\mathrm{N}_{2} \mathrm{H}^{+}$and $\mathrm{H}_{2} \mathrm{D}^{+}$, causing the noise to be of about $95 \mathrm{mJy}^{\text {beam }}{ }^{-1}$ per $0.025 \mathrm{~km} \mathrm{~s}^{-1}$ velocity channel, despite flagging the antennas with the highest system temperature.

In this work, we focus on the $\mathrm{DCO}^{+} 3-2$ and $\mathrm{N}_{2} \mathrm{D}^{+}$ 3-2 lines from the Cycle $012 \mathrm{~m}$ array obsevations, in addition to $\mathrm{c}-\mathrm{C}_{3} \mathrm{H}_{2} 6_{0,6}-5_{1,5}, \mathrm{DCO}^{+} 5-4, \mathrm{~N}_{2} \mathrm{H}^{+} 5-4$, and $\mathrm{H}_{2} \mathrm{D}^{+} 1_{1,0}-$ $1_{1,1} 12 \mathrm{~m}$ array observations from Cycle 2. Line transitions and frequencies, together with peak intensities and line widths are listed in Table 1.

Additionally, single-dish APEX observations in the ON/OFF mode were carried out on 22 and 24 October, 2016 using the heterodyne instrument SheFI (Belitsky et al. 2006; Vassilev et al. 2008) with Bands APEX-1 (213-275 GHz) and APEX-2 (267-378 GHz), targeting $\mathrm{DCO}^{+} 3-2$ and 5-4, as well as $\mathrm{C}_{2} \mathrm{H}$ 4-3. These observations were taken to compare the location of $\mathrm{C}_{2} \mathrm{H}$ in both VLA 1623 and IRAS 16293, as well as to have a separate verification and comparison of the physical parameters derived from ALMA observations and single-dish. Several transitions of NO and $\mathrm{HCN}$ were detected, both of which can form in gas and surface reactions, whereas $\mathrm{N}_{2} \mathrm{H}^{+}$ and $\mathrm{N}_{2} \mathrm{D}^{+}$only form in the gas. $\mathrm{NO}$ and $\mathrm{HCN}$ are not analyzed any further in this work. The observations were centered on VLA 1623 A $\left(\alpha_{J 2000}=16: 26: 26.390 ; \delta_{J 2000}=-24: 24: 30.688\right)$. The typical RMS noise was $100 \mathrm{mK}$ for APEX-1 and between 50 and $80 \mathrm{mK}$ for APEX-2 in $0.1 \mathrm{~km} \mathrm{~s}^{-1}$ channels. Peak temperatures $\left(T_{\mathrm{mb}}\right)$ and line widths for Gaussian fits to the single dish lines are listed in Table 1. The typical calibration uncertainties are about $10 \%$ for the APEX SheFI instruments in the 230 and $345 \mathrm{GHz}$ Bands. For APEX-1 and APEX-2 observations, the HPBW is $28.7^{\prime \prime}$ and $18^{\prime \prime}$, respectively. The main beam efficiencies used are $\eta_{\mathrm{mb}}=0.75$ at $230 \mathrm{GHz}$, and $\eta_{\mathrm{mb}}=0.73$ at $345 \mathrm{GHz}$.

\section{Results}

\subsection{IRAS 16293-2422}

The molecules $\mathrm{c}-\mathrm{C}_{3} \mathrm{H}_{2}, \mathrm{C}_{2} \mathrm{H}$, and $\mathrm{DCO}^{+}$from the PILS spectral survey (Jørgensen et al. 2016) are considered here, together with $\mathrm{DCO}^{+}$and $\mathrm{N}_{2} \mathrm{D}^{+}$from the SMA spectral survey (Jørgensen et al. 2011). The PILS survey images are obtained from the combined $12 \mathrm{~m}$ array and ACA, which picks up the small and large scale emission from scales less than $13^{\prime \prime}$. The peak intensities 


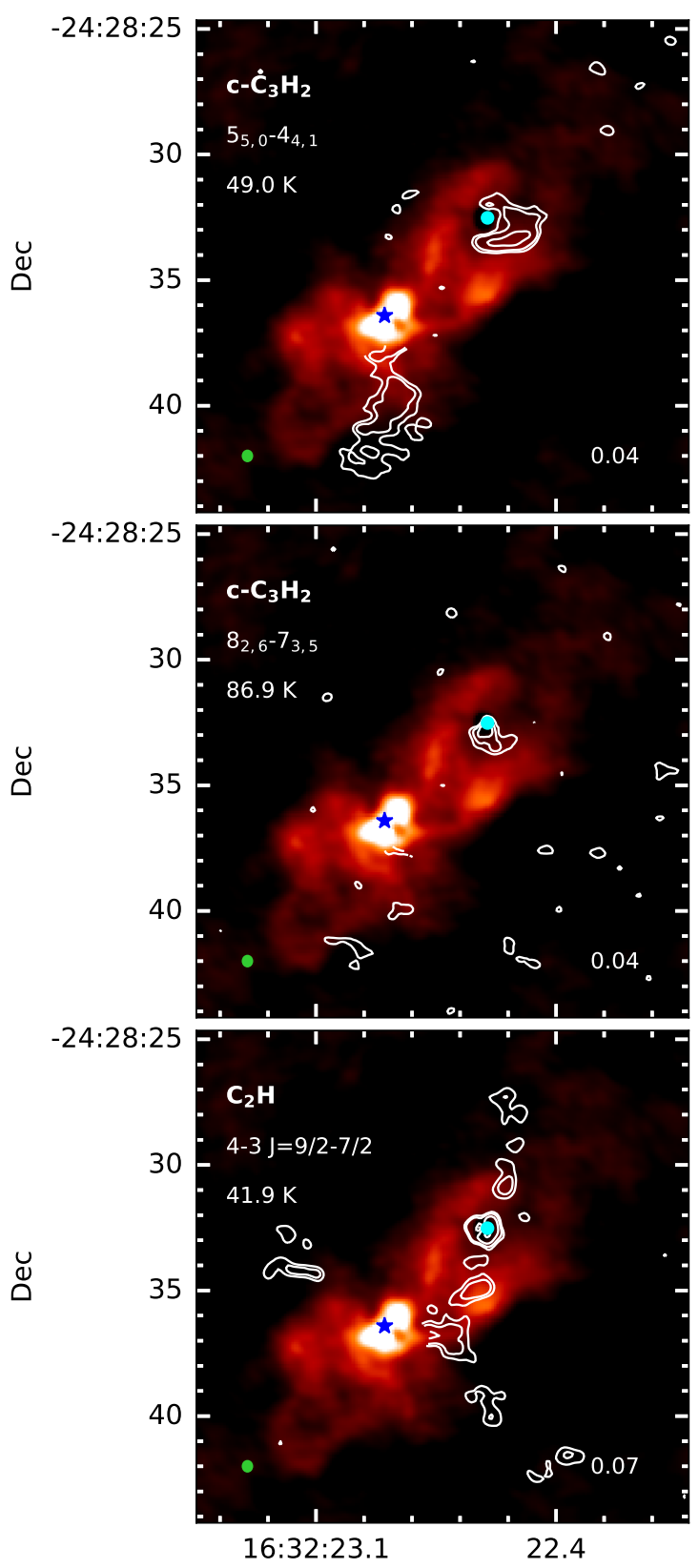

RA

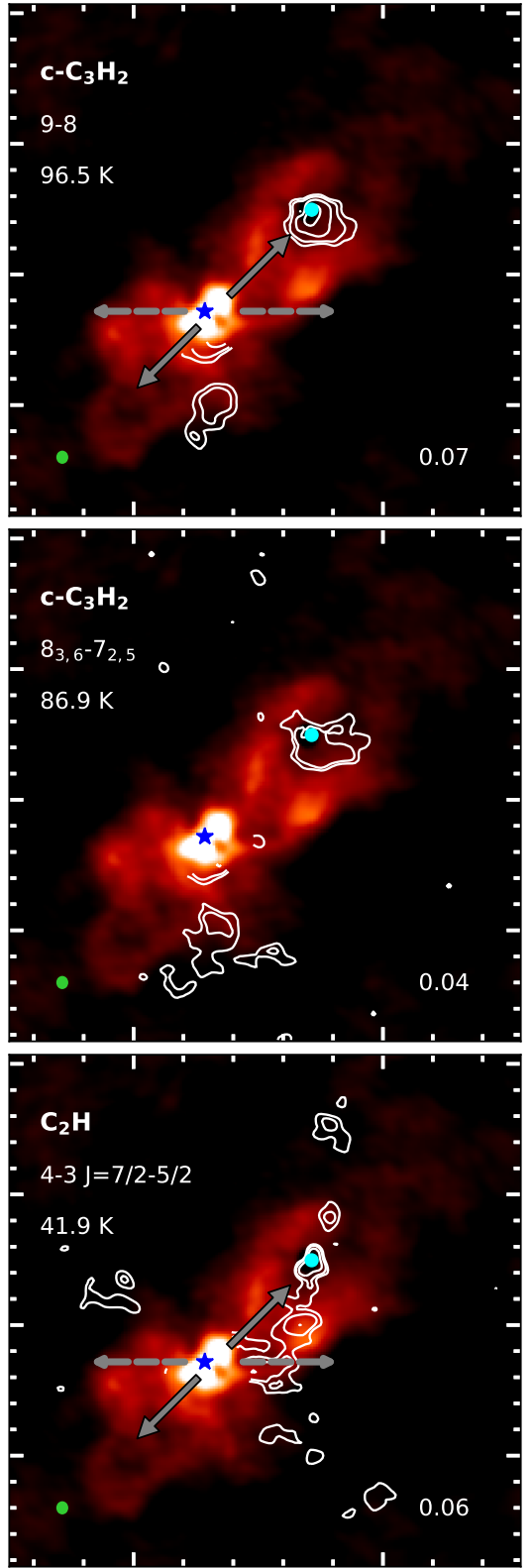

$16: 32: 23.1$
22.4

RA

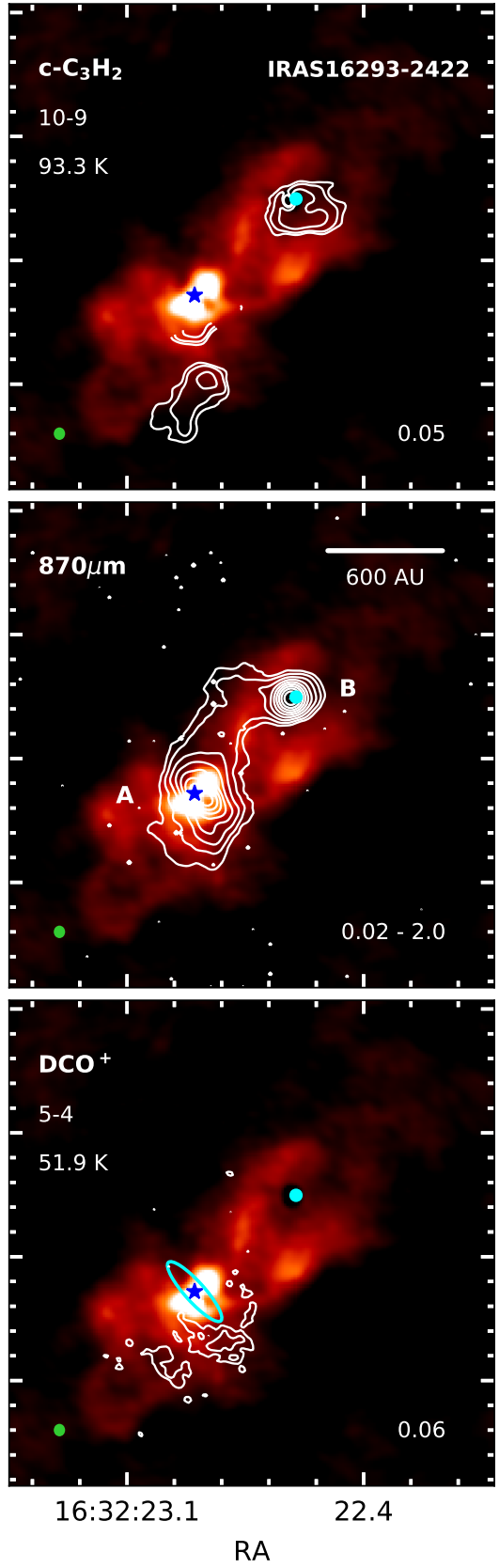

RA

Fig. 2. Intensity integrated maps (contours) of $\mathrm{c}-\mathrm{C}_{3} \mathrm{H}_{2}, \mathrm{C}_{2} \mathrm{H}, \mathrm{DCO}^{+}$, and continuum toward IRAS 16293-2422. Intensity integrated $\mathrm{H}_{2} \mathrm{CO} 5-4$ $\left(0-60 \mathrm{~km} \mathrm{~s}^{-1}\right)$ is shown in color scale. Contours show the respective lines in steps of 2, 3, 5, 20, and $60 \sigma$, with $\sigma\left(\mathrm{Jy} \mathrm{beam}^{-1} \mathrm{~km} \mathrm{~s}^{-1}\right)$ indicated in the lower right of each panel. For continuum at $870 \mu \mathrm{m}$, the levels are logarithmically spaced between 0.02 and $2 \mathrm{Jy}$ beam ${ }^{-1}$, and highlight the ridge that spans between sources A and B. The positions of IRAS 16293-2422 A and B are indicated with a star and circle, respectively. The gray arrows indicate the outflow directions, while the cyan ellipse shows the disk-like structure. The green circle on the bottom left panel indicates the beam of the combined $12 \mathrm{~m}$ and ACA observations. For the $\mathrm{c}-\mathrm{C}_{3} \mathrm{H}_{2}$ and $\mathrm{C}_{2} \mathrm{H}$ panels, the emission centered on $\mathrm{A}$ is contamination from other molecule(s) and is masked out in a radius of $2^{\prime \prime}$ from the position of A.

and widths of each line are listed in Table 1 . Intensity integrated maps of each line overlaid on $\mathrm{H}_{2} \mathrm{CO}$ are shown in Fig. 2. $\mathrm{H}_{2} \mathrm{CO}$ $5_{1,5}-4_{1,4}$ from the PILS survey (van der Wiel et al., in prep.) is used as a reference for the more extended envelope and one of the outflow directions. The nominal velocities at which most species emit at sources $\mathrm{A}$ and $\mathrm{B}$ are $V_{\mathrm{LSR}}=3.2$ and $2.7 \mathrm{~km} \mathrm{~s}^{-1}$. $\mathrm{c}-\mathrm{C}_{3} \mathrm{H}_{2}$ and $\mathrm{C}_{2} \mathrm{H}$ spectra at selected positions are presented in Figs. 3 and B.1.

In the 5-4 (PILS) and 3-2 (SMA) transitions $\mathrm{DCO}^{+}$ is detected, with a half-crescent shape centered around source A (Figs. 2 and 4). The peak is red-shifted and located $\sim 2^{\prime \prime}$ southwest of source A in both transitions, consistent with the red-shifted emission of the disk-like structure to the southwest (Oya et al. 2016). Weak absorption is detected toward source B in the PILS observations, which is consistent with previous studies that indicate infall motions through an inverse P-Cygni profile (Zapata et al. 2013). The $\mathrm{DCO}^{+}$emission south of source $\mathrm{A}$ is weak, peaking at $3 \sigma$ in the 5-4 transition and at $5 \sigma$ in the 3-2 transition. It is slightly extended to the south along the outflow, but not as far as $\mathrm{c}-\mathrm{C}_{3} \mathrm{H}_{2}$. In agreement with previous observations of $\mathrm{DCO}^{+}$and $\mathrm{c}-\mathrm{C}_{3} \mathrm{H}_{2}$ for other objects (Spezzano et al. 2016a,b), these two molecules are spatially anti-correlated. 

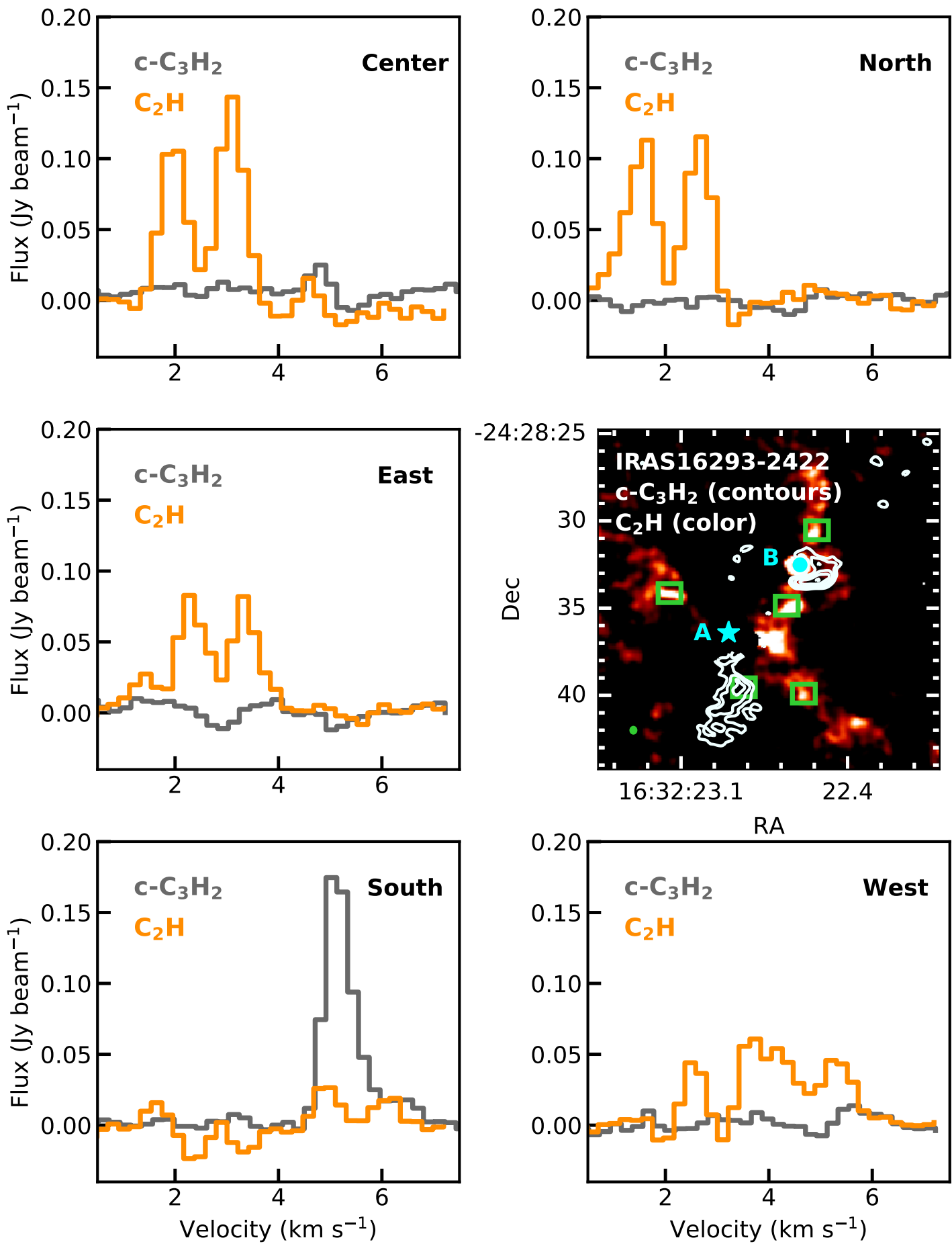

Fig. 3. IRAS $16293-2422 \mathrm{c}-\mathrm{C}_{3} \mathrm{H}_{2} 5-4$ and $\mathrm{C}_{2} \mathrm{H} 4-3 \quad J=9 / 2-7 / 2$ spectra taken at five positions, indicated by the green boxes in the intensity integrated map presented in the center right panel, while the green circle on the bottom left panel indicates the beam of the combined $12 \mathrm{~m}$ and ACA observations. The anti-correlation of both molecules is seen at all positions. The region within a radius of $2^{\prime \prime}$ from the position of source A is contaminated by other molecular species, and is masked out for these maps.

Five narrow $\left(F W H M \approx 1 \mathrm{~km} \mathrm{~s}^{-1}\right)$ lines of $\mathrm{c}-\mathrm{C}_{3} \mathrm{H}_{2}$ in the $349-352 \mathrm{GHz}$ frequency range with $E_{\text {up }}$ ranging from 48 to $96 \mathrm{~K}$ are studied in this work. The emission peaks to the south of A, seen clearly in the top row of Fig. 2. The lines are also seen near source B, at one ALMA beam offset from the source. The southern emission extends from the circumstellar region of source A, and peaks at $\leq 5 \sigma$ about $\sim 4^{\prime \prime}$ away from the source position. Toward source A itself, the spectrum is too confused to identify the separate molecular lines. Thus, the region is masked out in the maps within $2^{\prime \prime}$ from the source position. Comparing c- $\mathrm{C}_{3} \mathrm{H}_{2}$ with $\mathrm{H}_{2} \mathrm{CO}$ (Fig. 2) suggests that it could arise from one side of the southern outflow cavity wall. The asymmetric heating of the outflow cavity could be due to the behavior of the outflow from IRAS 16293 A. Observations of the outflow of IRAS 16293 A at large and small scales suggest that the outflow has shifted direction from eastwest (dashed arrow in Fig. 1) to southeast-northwest (solid arrow in Fig. 1). The shift of outflow direction could have swept up material, thus causing the 


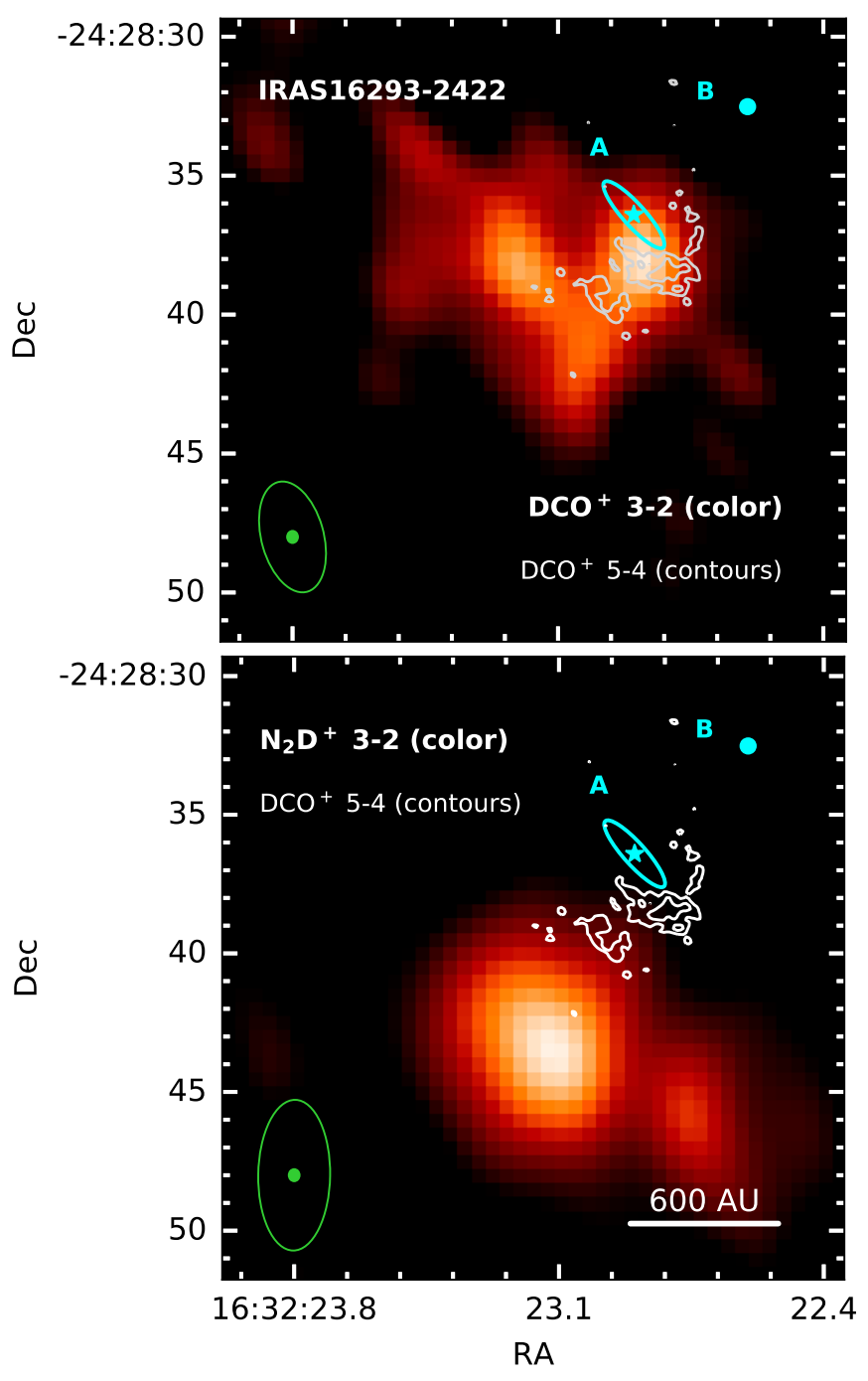

Fig. 4. Intensity integrated maps of $\mathrm{DCO}^{+} 3-2$ (top panel) and $\mathrm{N}_{2} \mathrm{D}^{+}$ 3-2 (bottom panel) observed with the SMA, overlaid with $\mathrm{DCO}^{+}$ 5-4 (contours) observed with ALMA (combined $12 \mathrm{~m}$ array and ACA). Contours are the same as in Fig. 2 with $\sigma=0.06 \mathrm{Jy} \mathrm{beam}^{-1}$. The positions of IRAS 16293-2422 A and B are indicated with a star and circle, respectively. The cyan ellipse shows the disk-like structure. The filled green ellipses show the beam for the ALMA observations, while the unfilled ellipse shows the beam for the SMA observations. Both $\mathrm{DCO}^{+}$ transitions match spatially, and $\mathrm{N}_{2} \mathrm{D}^{+}$is located beyond the extent of the $\mathrm{DCO}^{+}$emission. We note that this figure has a different center from that of Fig. 2.

asymmetric morphology of the outflow cavity, and consequently $\mathrm{c}-\mathrm{C}_{3} \mathrm{H}_{2}$ to only be present on one side of the outflow cavity. The emission around source B may either be from the circumstellar region or the outflow cavity, but due to the orientation it is difficult to say.

We clearly detect $\mathrm{C}_{2} \mathrm{H}$ in both spin doubling transitions with each transition showing a characteristic double hyperfine structure pattern. The emission within $2^{\prime \prime}$ of source $\mathrm{A}$ is masked out due to contamination from other molecular species. $\mathrm{C}_{2} \mathrm{H}$ emission is located in a filament-like structure extending from north to south, passing through source B (Fig. 2). A second, weaker structure formed by a string of clumps extends from northeast to southwest, apparently passing through source $\mathrm{A}$. $\mathrm{C}_{2} \mathrm{H}$ is diffuse and weak, peaking at $\lesssim 5 \sigma$ on all off-source positions on the map (Fig. 3). The emission around source B is brighter, peaking at $10 \sigma$ in the intensity integrated map. From the channel map, the emission appears to have a subtle velocity gradient from north to south at source $\mathrm{B}$. However, the $\mathrm{C}_{2} \mathrm{H}$ emission does not match the structure and extent of $\mathrm{c}-\mathrm{C}_{3} \mathrm{H}_{2}$ (or $\mathrm{H}_{2} \mathrm{CO}$ ) in either transition (Figs. 3 and B.1). It should be noted that neither $\mathrm{C}_{2} \mathrm{H}$ nor $\mathrm{c}-\mathrm{C}_{3} \mathrm{H}_{2}$ coincide with the dust ridge seen in the continuum emission (Fig. 2; see also Jacobsen et al. 2018).

The molecule $\mathrm{N}_{2} \mathrm{D}^{+} 3-2$ is mostly resolved out in the PILS survey, but it is detected southeast of $\mathrm{DCO}^{+}$with the SMA (Fig. 4), located $7^{\prime \prime}$ away from the continuum position of source A with a $S / N=7$ (Jørgensen et al. 2011). Similar to $\mathrm{DCO}^{+}$, there is no $\mathrm{N}_{2} \mathrm{D}^{+}$emission toward $\mathrm{B}$. No transition of $\mathrm{N}_{2} \mathrm{D}^{+}$was covered in the Band 7 observations.

\subsection{VLA 1623-2417}

Two transitions of $\mathrm{DCO}^{+}$and one transition each of $\mathrm{c}-\mathrm{C}_{3} \mathrm{H}_{2}$, $\mathrm{N}_{2} \mathrm{H}^{+}$, and $\mathrm{N}_{2} \mathrm{D}^{+}$were observed with ALMA in Bands 6 and 7. Additional APEX observations detected two transitions of $\mathrm{DCO}^{+}$and $\mathrm{C}_{2} \mathrm{H}$. Intensities and line widths are listed in Table 1. Figure 5 shows the intensity integrated maps for the ALMA observations. All of these molecules trace material associated with VLA 1623 A, but not the other two components of the system, VLA $1623 \mathrm{~B}$ and $\mathrm{W}$.

The $\mathrm{DCO}^{+} 3-2$ ALMA $12 \mathrm{~m}$ array and ACA observations have been separately analyzed in detail in Murillo et al. (2015). The 3-2 ACA map shows a smooth distribution peaking southwest of the source, with the blue-shifted emission extending northeast, but no clear red-shifted counterpart southwest. Here, we present additional ALMA Band $712 \mathrm{~m}$ array observations of $\mathrm{DCO}^{+} 5-4$. In both transitions of $\mathrm{DCO}^{+}$, the red-shifted emission, located to the south of VLA $1623 \mathrm{~A}$, is clearly seen and is stronger than the blue-shifted emission located to the north. The $\mathrm{DCO}^{+} 5-4$ emission is three times stronger than the 3-2 emission with the $12 \mathrm{~m}$ array, which makes the blue-shifted emission clearly visible. For both transitions, the velocity gradients are consistent. The $\mathrm{DCO}^{+} 3-2$ emission borders, and the disk structure observed to be driven by VLA $1623 \mathrm{~A}$ are relatively compact. Even more interesting, however, is that $\mathrm{DCO}^{+}$in the 5-4 transition extends closer to the position of VLA 1623 A than in the 3-2 transition (Fig. 5).

The molecule $\mathrm{DCO}^{+}$forms at temperatures below $20 \mathrm{~K}$, where $\mathrm{CO}$ freezes out. The position of the $\mathrm{DCO}^{+} 3-2$ peak along the disk plane was found to be the product of diskshadowing, which causes a temperature drop at the edge of the disk, whereas along the outflow direction no such effect was observed (Murillo et al. 2015). The APEX observations of $\mathrm{DCO}^{+}$in both transitions show a single peak at the systemic velocity $\left(3.7-4 \mathrm{~km} \mathrm{~s}^{-1}\right)$ and a peak intensity of $3.6 \mathrm{~K}$ for the 3-2 transition, the same as obtained from JCMT observations by Jørgensen et al. (2004). The beamsize for the APEX-1 and 2 Bands covers approximately the full extent of the $\mathrm{DCO}^{+}$emission seen in the ACA map. For the $\mathrm{DCO}^{+} 3-2$ transition, the ALMA observations recover about $28 \%$ of the flux detected in the APEX observations (117.1 $\mathrm{Jy} \mathrm{km} \mathrm{s}^{-1}$ with $24 \mathrm{Jy} \mathrm{K}^{-1}$ ), while $20 \%$ was recovered with the $\mathrm{DCO}^{+} 5-4$ ALMA observations (APEX: $53.7 \mathrm{Jy} \mathrm{km} \mathrm{s}^{-1}$ with $24 \mathrm{Jy} \mathrm{K}^{-1}$ ).

One low-lying transition of $\mathrm{c}-\mathrm{C}_{3} \mathrm{H}_{2}$ is detected with the short baselines of the $12 \mathrm{~m}$ array, but not the long baselines. The detection of $\mathrm{c}-\mathrm{C}_{3} \mathrm{H}_{2}$ with only the short baselines of the $12 \mathrm{~m}$ array indicates that the emission is extended without a compact structure component. The $\mathrm{c}-\mathrm{C}_{3} \mathrm{H}_{2}$ emission is oriented perpendicular to the disk and seems to trace the cavity of the outflow driven by VLA 1623 A out to $3^{\prime \prime}$ from the source position. There is no detection of $\mathrm{c}-\mathrm{C}_{3} \mathrm{H}_{2}$ emission in the disk traced by $\mathrm{C}^{18} \mathrm{O}$ or at 

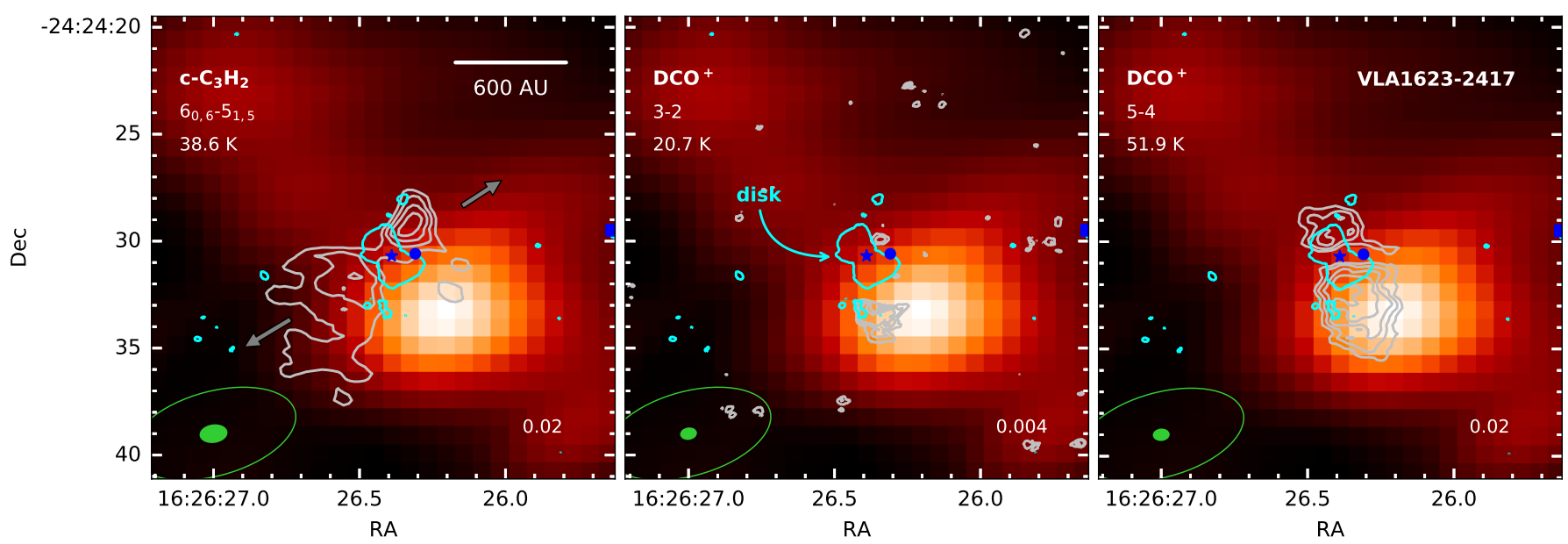

Fig. 5. Intensity integrated maps of (white contours) $\mathrm{c}-\mathrm{C}_{3} \mathrm{H}_{2}$ and $\mathrm{DCO}^{+}(12 \mathrm{~m}$ array) toward VLA 1623-2417. DCO $3-2$ from ACA observations is shown in color scale. The gray arrows on the left panel show the outflow direction. The cyan line is the $3 \sigma$ contour of $\mathrm{C}^{18} \mathrm{O}$ in order to show the location and extent of the rotating disk centered on VLA 1623-2417 A. Gray contours show the respective lines in steps of 3, 4, 5 and $6 \sigma$, except for $\mathrm{DCO}^{+} 3-2$ where the contours start at $4 \sigma$. The value of $\sigma\left(\mathrm{Jy} \mathrm{beam}^{-1} \mathrm{~km} \mathrm{~s}^{-1}\right)$ is indicated in the lower right of each panel. The positions of VLA 1623-2417 A, B, and W are indicated with a star, circle, and square, respectively. The filled green ellipses show the beam for the $12 \mathrm{~m}$ array observations (contours), while the unfilled ellipse shows the beam of the ACA observation (color scale).

the disk-envelope interface, down to the noise level. The material along the outflow cavity exhibits signatures of rotation, most notable in the southeast lobe, with a velocity range and gradient direction similar to that of $\mathrm{DCO}^{+}$and $\mathrm{C}^{18} \mathrm{O}$ (Murillo et al. 2013). However, treating the kinematics of the outflow cavity wall traced by $\mathrm{c}-\mathrm{C}_{3} \mathrm{H}_{2}$ is beyond the scope of this paper, and will be presented in a separate paper.

We detect $\mathrm{C}_{2} \mathrm{H}$ with APEX, with the hyperfine components of each transition being clearly distinguished (Fig. 6). Both transitions are located at the systemic velocity of VLA 1623 A (3.7-4.0 $\mathrm{km} \mathrm{s}^{-1}$ ) and show no broadening, indicating that the emission is most likely related to the envelope material of VLA 1623 A.

In our ALMA observations $\mathrm{N}_{2} \mathrm{H}^{+}$and $\mathrm{N}_{2} \mathrm{D}^{+}$are not detected. Possible reasons for this could be either due to the emission being very extended and thus resolved out in the interferometric observations, or the abundance of these molecules being too low to be detected. This is a surprising contrast to several other young embedded Class 0 sources which do show $\mathrm{N}_{2} \mathrm{H}^{+}$ and $\mathrm{N}_{2} \mathrm{D}^{+}$(Jørgensen et al. 2004; Tobin et al. 2013). It is also interesting to note the lack of molecular line emission toward VLA 1623 in contrast to IRAS 16293 (Fig. 7). The nondetections of $\mathrm{N}_{2} \mathrm{H}^{+}$and $\mathrm{N}_{2} \mathrm{D}^{+}$are further analyzed in Sect. 4.4. $\mathrm{H}_{2} \mathrm{D}^{+}$ is also not detected in our Cycle 2 Band 7 observations, this is consistent with the JCMT observations reported by Friesen et al. (2014). $\mathrm{H}_{2} \mathrm{D}^{+}$is not further treated in this work.

\section{Analysis}

$\mathrm{DCO}^{+}$appears to peak offset from the protostellar positions bordering the disk-like structures in both sources (Figs. 2 and 5). Here, we analyze the peak position first through chemical modeling of the observed emission (Sect. 4.1.1), and then using the line ratios to constrain the physical structure (temperature, density) and the associated chemistry (Sect. 4.1.2).

To study the physical conditions of the region traced by $\mathrm{c}-\mathrm{C}_{3} \mathrm{H}_{2}$, line ratios of the detected transitions are used (Sect. 4.2). This is combined with $\mathrm{C}_{2} \mathrm{H}$ to obtain the $\mathrm{c}-\mathrm{C}_{3} \mathrm{H}_{2} / \mathrm{C}_{2} \mathrm{H}$ abundance ratio and show how the ratio varies with position (Sect. 4.3). $\mathrm{c}-\mathrm{C}_{3} \mathrm{H}_{2}$ and $\mathrm{C}_{2} \mathrm{H}$ can be produced by the destruction of large hydrocarbons through UV irradiation (top-down chemistry), or through the accumulation of $\mathrm{C}$ and $\mathrm{H}$ atoms to form small hydrocarbons (bottom-up). Because of the many different destruction and formation pathways, chemical modeling of these two molecules is not included in this work.

\section{1. $D C O^{+}$}

\subsection{1. $\mathrm{DCO}^{+}$distribution}

In Murillo et al. (2015), the distribution of $\mathrm{DCO}^{+}$around VLA 1623 was found to be altered by the presence of a rotationally supported disk, causing the emission to shift inward along the disk plane but not along other directions. In this section, the $\mathrm{DCO}^{+}$emission around IRAS 16293 is modeled, aiming to find whether the distribution of $\mathrm{DCO}^{+}$in IRAS 16293 is product of the same phenomenon as observed in VLA 1623.

The $\mathrm{DCO}^{+}$chemistry is particularly sensitive to temperature. To model the observed emission for IRAS 16293 and VLA 1623, a simple steady state, analytic chemical network that accounts for the basic reactions leading to the production and destruction of $\mathrm{DCO}^{+}$is used.

Since $\mathrm{CO}$ and $\mathrm{H}_{2} \mathrm{D}^{+}$are the precursors of $\mathrm{DCO}^{+}$, the production of $\mathrm{H}_{2} \mathrm{D}^{+}$will be the rate-determining reaction in the chemical network, since it will dictate the production of $\mathrm{DCO}^{+}$. The $\mathrm{H}_{2} \mathrm{D}^{+}$production and destruction reaction is given by

$\mathrm{H}_{3}^{+}+\mathrm{HD} \underset{\Delta E}{\rightleftharpoons} \mathrm{H}_{2} \mathrm{D}^{+}+\mathrm{H}_{2}$,

where the activation energy $\Delta E \sim 220 \mathrm{~K}$ in the back reaction is due to the difference in zero-point energy. A crucial factor for deuterium chemistry is the ortho-to-para ratio of $\mathrm{H}_{2}$ (Flower et al. 2006; Pagani et al. 2009b). This is included in the back reaction of the chemical network (Eq. (1)), since it is here where the distinction has the most significant effect (Murillo et al. 2015). The reactions and parameters for $o-\mathrm{H}_{2}$ and $o-\mathrm{H}_{2}$ were adapted from Walmsley et al. (2004). The ortho-to-para ratio is set to have a lower limit of $10^{-3}$ at low temperatures, as constrained from observations and models (Flower et al. 2006). The rate coefficient for the two-body reaction is expressed as

$k=\alpha\left(\frac{T}{300}\right)^{\beta} \exp \left(-\frac{\gamma}{T}\right) \mathrm{cm}^{3} \mathrm{~s}^{-1}$, 


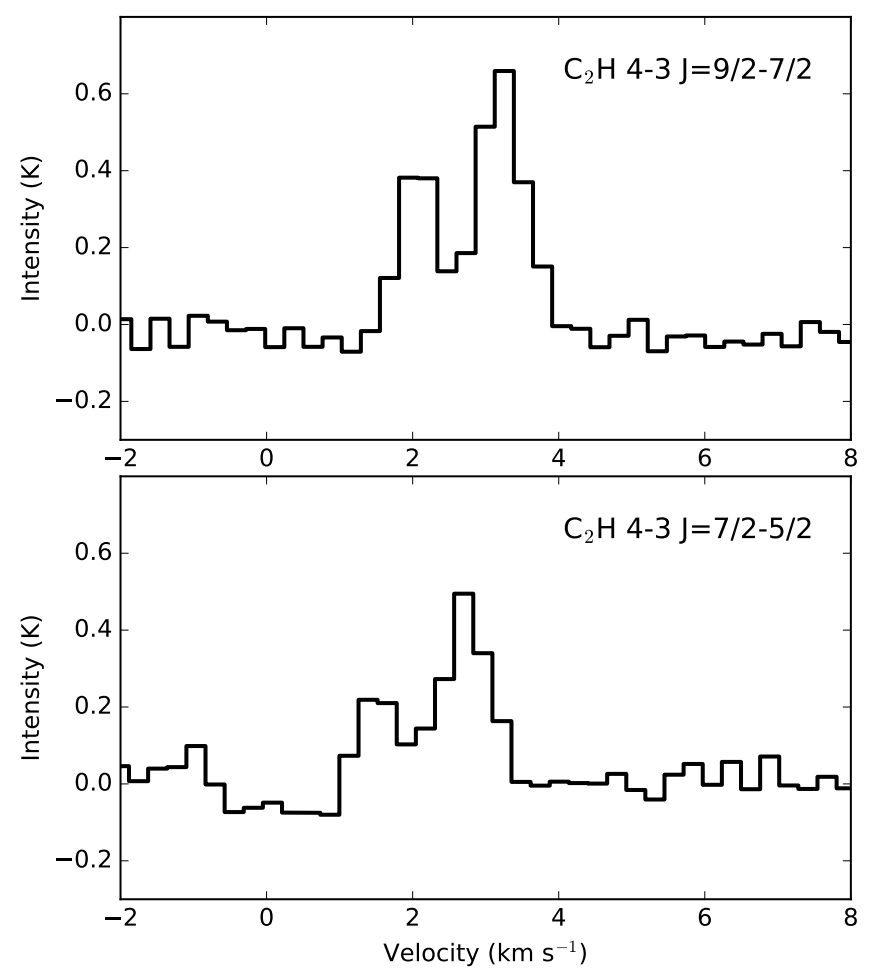

Fig. 6. Single pointing APEX observations of $\mathrm{C}_{2} \mathrm{H}$ centered on VLA $1623-2417$ A.

where $T$ is the temperature of the gas. For cosmic ray ionization, important in the generation of $\mathrm{H}_{3}^{+}$, the rate coefficient is given by

$k=\zeta \mathrm{s}^{-1}$

where $\zeta=1.26 \times 10^{-17}$ is the cosmic-ray ionization rate of $\mathrm{H}_{2}$. The reactions and rate coefficients used in this work are the same as those listed in Murillo et al. (2015).

Since $\mathrm{CO}$ is a parent molecule of $\mathrm{DCO}^{+}$, its abundance will impact the production of $\mathrm{DCO}^{+}$. The profile of the $\mathrm{CO}$ abundance is taken to be either constant or with a drop used to simulate freeze-out. The drop is set by the $\mathrm{CO}$ sublimation temperature $T_{\text {sub }}$ and desorption density $n_{\mathrm{de}}$. These limits dictate the boundary where $\mathrm{CO}$ is in the gas phase $\left(T>T_{\text {sub }}\right)$ or freezes onto the dust grains $\left(T<T_{\mathrm{sub}}\right)$; and when the freeze-out time-scales for $\mathrm{CO}$ are too long $\left(n<n_{\mathrm{de}}\right)$ compared to the lifetime of the core (Jørgensen et al. 2005c). The results of the chemical modeling are passed through RATRAN (Hogerheijde \& van der Tak 2000), and then synthetic data cubes are generated in order to directly compare with the observations. Because the $\mathrm{DCO}^{+}$emission is weak, the radial profile of the observations is obtained by using a cut with a width that covers the red-shifted peak. The model radial profile, on the other hand, is obtained from a simple cut to the synthetic data cubes since the models are symmetric flat disks. The network and further details of the model and post-processing are given in Murillo et al. (2015).

The model requires a density and temperature profile of the source as a function of radius. For IRAS 16293, the power-law density and temperature profile from Crimier et al. (2010) is adopted. Two assumptions are made, namely, the density and temperature profile is centered on IRAS 16293 A and that it is the main contributor to the luminosity of the core, consistent with the recent analysis of Jacobsen et al. (2018). Crimier

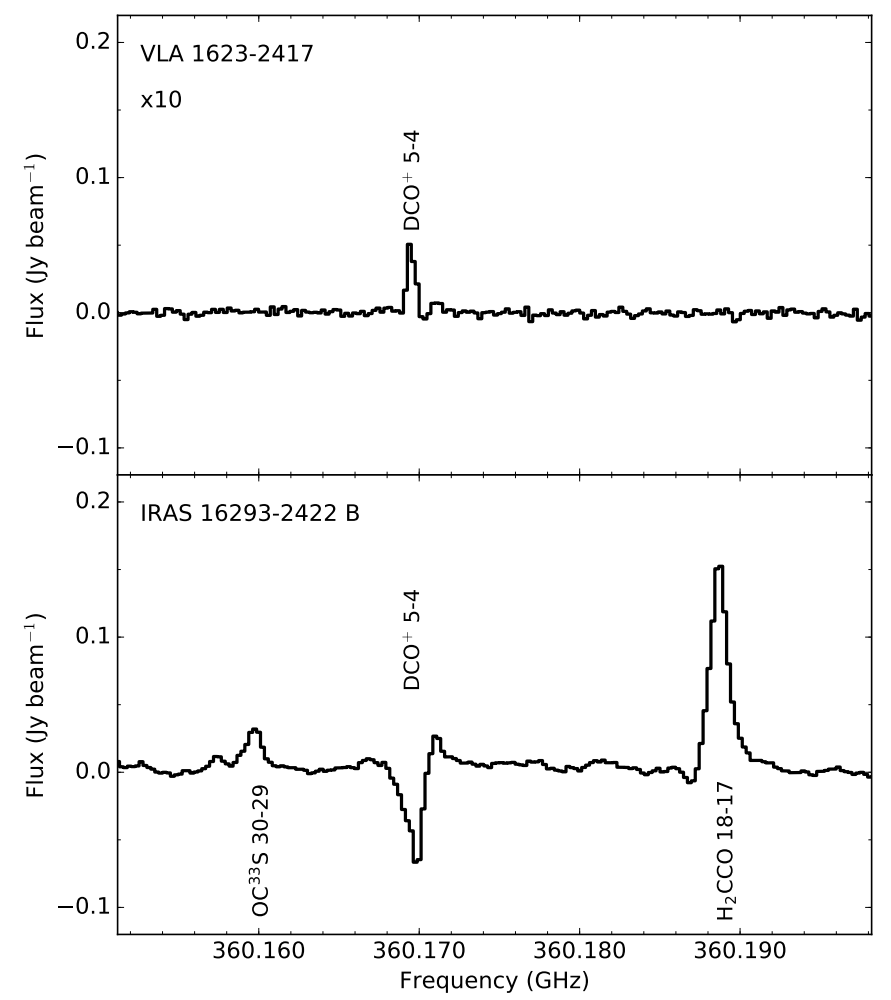

Fig. 7. Comparison of the spectra centered on $\mathrm{DCO}^{+} 5-4$ of VLA $1623-2417$ A (12 $\mathrm{m}$ array) and one beam offset from IRAS 16293-2422 B (combined $12 \mathrm{~m}$ array and ACA). The spectra for VLA $1623-2417$ has been multiplied by a factor of 10 in order to compare more easily. We note the lack of molecular line emission toward VLA 1623-2417 in contrast to IRAS 16293-2422.

et al. (2010) also showed that the emission is dominated by one of the two components, most likely IRAS 16293 A, rather than being centered between the two sources. Thus, our assumptions should not introduce major issues in our modeling. While several physical profiles have been derived for IRAS 16293 (e.g., Schöier et al. 2002), only one is adopted here since we alter the density and temperature profiles by an arbitrary factor, exploring the effects of these parameters on the production of $\mathrm{DCO}^{+}$. For VLA 1623, we adopt the power-law density and temperature profile from Jørgensen et al. (2002). Here again we assume the density and temperature profile is centered and dominated by VLA 1623 A. Given that VLA 1623 B does not contribute much to the line emission nor the continuum, and that VLA $1623 \mathrm{~W}$ is $10^{\prime \prime}$ away, this should not produce issues in the resulting model. The temperature and density profile for VLA 1623 is also altered by an arbitrary factor to study the effect on $\mathrm{DCO}^{+}$production. The variations in the temperature and density profiles used in this work are listed in Table 2 for both systems.

For the $\mathrm{DCO}^{+}$models, we explore the parameter ranges of $T_{\text {sub }}=20-40 \mathrm{~K}, n_{\mathrm{de}}=10^{5}-10^{8} \mathrm{~cm}^{-3}$, and $X[\mathrm{CO}]=10^{-7}-10^{-4}$. The parameters for the best by-eye approximation to the observed $\mathrm{DCO}^{+}$peak position are listed in Table 3 for both systems. The best approximated model of the $\mathrm{DCO}^{+} 3-2$ emission around VLA 1623 (Murillo et al. 2015) are reproduced here and compared with the results of $\mathrm{DCO}^{+}$toward IRAS 16293 .

For both systems, we find that the constant $\mathrm{CO}$ abundance profile produces a $\mathrm{DCO}^{+}$peak further out than where it is observed (gray dashed line in fourth and fifth rows of Fig. 8), and the peak position does not shift with a change in the abundance 
Table 2. Tests of the temperature and density profiles of the two sources.

\begin{tabular}{cccccccc}
\hline \hline & \multicolumn{2}{c}{ IRAS $16293-2422($ Crimier et al. 2010) } & & \multicolumn{3}{c}{ VLA $1623-2417(\mathrm{~J} \varnothing r g e n s e n$ et al. 2002) } \\
\cline { 2 - 3 } \cline { 6 - 7 } Test & $T_{27 \mathrm{AU}}(\mathrm{K})$ & $n_{27 \mathrm{AU}}\left(\mathrm{cm}^{-3}\right)$ & Note & & $T_{4 \mathrm{AU}}(\mathrm{K})$ & $n_{4 \mathrm{AU}}\left(\mathrm{cm}^{-3}\right)$ & Note \\
\hline 1 & 300.0 & $2.36 \times 10^{9}$ & unchanged & & 250.0 & $1.62 \times 10^{9}$ & unchanged \\
2 & 300.0 & $2.36 \times 10^{10}$ & $n$ increased by 10 & & 250.0 & $1.62 \times 10^{10}$ & $n$ increased by 10 \\
3 & 300.0 & $2.36 \times 10^{8}$ & $n$ decreased by 10 & & 250.0 & $1.62 \times 10^{8}$ & $n$ decreased by 10 \\
4 & 100.0 & $2.36 \times 10^{9}$ & $T$ decreased by 3 & & 166.7 & $1.62 \times 10^{9}$ & $T$ decreased by 1.5 \\
\hline
\end{tabular}

Table 3. $\mathrm{DCO}^{+}$best approximation model parameters.

\begin{tabular}{ccc}
\hline \hline Parameter & IRAS $16293-2422$ & VLA $1623-2417$ \\
\hline$T_{\text {peak }}(\mathrm{K})$ & $17-19$ & $11-16$ \\
Drop boundaries: & & \\
$T_{\text {sub }}(\mathrm{K})$ & 35 & 35 \\
$n_{\text {de }}\left(\mathrm{cm}^{-3}\right)$ & $\leq 10^{6}$ & $3 \times 10^{6}$ \\
CO abundance: & & \\
Inner $X_{\text {in }}$ & $10^{-5}$ & $10^{-5}$ \\
Drop $X_{\mathrm{D}}$ & $10^{-6}$ & $10^{-7}$ \\
Outer $X_{0}$ & $10^{-4}$ & $10^{-4}$ \\
\hline
\end{tabular}

(Murillo et al. 2015). The drop CO abundance profile produces a peak within the drop boundaries, $T_{\text {sub }}$ and $n_{\mathrm{de}}$. Altering these parameters changes the shape but not the position of the $\mathrm{DCO}^{+}$ peak (see Murillo et al. 2015).

Since the chemical conditions do not alter the peak position, the physical structure is examined. The original source density and temperature profiles for both sources also do not reproduce the position of the $\mathrm{DCO}^{+}$peak. Increasing or decreasing the density by one order of magnitude causes the $\mathrm{DCO}^{+}$peak to either shift outward or remain at a position similar to the unchanged density profile. Interestingly, only reducing the temperature profile by an arbitrary factor together with the drop $\mathrm{CO}$ abundance profile, causes the $\mathrm{DCO}^{+}$peak to shift inward for both systems (orange solid line in Fig. 8). A by-eye fit of the chemical model to the observations is used to constrain the decrease in the temperature profile (Fig. 8). The factor is constrained to be $1.5 \pm 0.2$ for VLA 1623 and $3.0 \pm 0.2$ for IRAS 16293. This is consistent with the results found for the $\mathrm{DCO}^{+}$ 3-2 toward VLA 1623 in Murillo et al. (2015), which explores the physical and chemical parameter space in more detail. Thus, the observed $\mathrm{DCO}^{+}$peak position is produced by a drop in the temperature along the plane perpendicular to the outflow(s). This drop in temperature can be caused by a structure, such as a disk, which shadows the outer regions, allowing the peak emission of molecules whose abundance is enhanced in cold gas to move inward.

It should be noted, however, that our simple chemical model cannot fully explain the inner part of the $\mathrm{DCO}^{+} 5-4$ emission observed toward VLA 1623 A and IRAS 16293 A (orange and black solid lines in the bottom row of Fig. 8). $\mathrm{DCO}^{+} 5-4$ emission at small radii could be located in the disk where both cold and warm chemical processes can contribute to its formation (Favre et al. 2015; Huang et al. 2017; Salinas et al. 2017). For warm $\mathrm{DCO}^{+}$to form, gas with temperatures up to $70 \mathrm{~K}$ (Favre et al. 2015) are needed. $\mathrm{DCO}^{+} 5-4$ is observed further into the disk of VLA 1623 A $\left(L_{\mathrm{bol}}=1 L_{\odot}\right.$; Murillo \& Lai 2013) than in IRAS $16293\left(L_{\mathrm{bol}}=18 L_{\odot}\right.$; Jacobsen et al. 2018) due to the lower temperature of the inner disk regions. Considering the original source profile which applies to the disk region (top row, solid red line, Fig. 8), IRAS 16293 A reaches gas temperatures of $70 \mathrm{~K}$ at about $1^{\prime \prime}$ or $120 \mathrm{AU}$, the outer part of the $200 \mathrm{AU}$ disk-like structure (Oya et al. 2016). In contrast, VLA 1623 A reaches $70 \mathrm{~K}$ at $0.1^{\prime \prime}$ or $12 \mathrm{AU}$ - in other words, significantly closer to the protostar.

Thus, the distribution of the cold $\mathrm{DCO}^{+}$around both VLA 1623 and IRAS 16293 is product of the presence of a disk(-like) structure, which causes a drop in temperature on the envelope gas at the edge of the disk(-like structure), that is, the disk-envelope interface. The presence of the disk(-like) structure generates an asymmetric temperature profile in the protostellar system.

\subsubsection{Line ratios and implied physical conditions}

Line ratios can provide an independent measure of the temperature of the region being traced by a molecule. The ratio of $\mathrm{DCO}^{+}$ 5-4/3-2 will provide an independent test of the results obtained with the chemical model of $\mathrm{DCO}^{+}$described in the previous section.

Using RADEX (van der Tak et al. 2007), we performed nonLTE excitation and radiative transfer calculations to constrain the temperature and density of the regions being traced by comparing the ratios of observed molecular lines with those calculated by the non-LTE excitation. We limit the range of $\mathrm{H}_{2}$ densities based on the source profile used for chemical modeling (Table 2 and Fig. 8) and the radial position of the emission being modeled. Using RADEX the column density of the emission was checked to see if it is produced by molecular line emission that is optically thin or thick at $\mathrm{H}_{2}$ densities of $1-8 \times 10^{7} \mathrm{~cm}^{-3}$ for IRAS 16293 and $2-6 \times 10^{6} \mathrm{~cm}^{-3}$ for VLA 1623 . For the bestfitting column densities of $(2-3) \times 10^{12} \mathrm{~cm}^{-2}$ and $\mathrm{H}_{2}$ densities of $10^{6}-10^{8} \mathrm{~cm}^{-3}$, the $\mathrm{DCO}^{+} 3-2$ and 5-4 emission is optically thin in both sources (Table 4). To produce optically thick lines, column densities of $>7 \times 10^{12} \mathrm{~cm}^{-2}$ would be needed for a temperature of $20 \mathrm{~K}$. As the emission of both lines are optically thin, the line intensity ratios are not affected by the adopted column density. All the molecular data files used in this work are obtained from the Leiden Atomic and Molecular Database (LAMDA; Schöier et al. 2005). The collisional rate coefficients for $\mathrm{DCO}^{+}$are based on the results of Botschwina et al. (1993) and Flower (1999). In order to compare the observed peak intensities with the results from RADEX, the observed peak intensities are converted from $\mathrm{Jy} \mathrm{beam}^{-1}$ to $\mathrm{K}$ using the relation $T_{\mathrm{mb}}=1.36 \lambda^{2} / \theta^{2} I_{v, \mathrm{obs}}$ where $\lambda$ is the wavelength in centimeters of the molecular transition, $\theta$ is the beam of the observations and $I_{v, \text { obs }}$ is the observed peak intensity in $\mathrm{mJy} \mathrm{beam}^{-1}$.

Here, we derive the physical parameters from the $\mathrm{DCO}^{+}$ 5-4/3-2 ratio for both sources. Figure 9 shows the variation of the $\mathrm{DCO}^{+} 5-4 / 3-2$ ratio with $\mathrm{H}_{2}$ density and temperature. For 

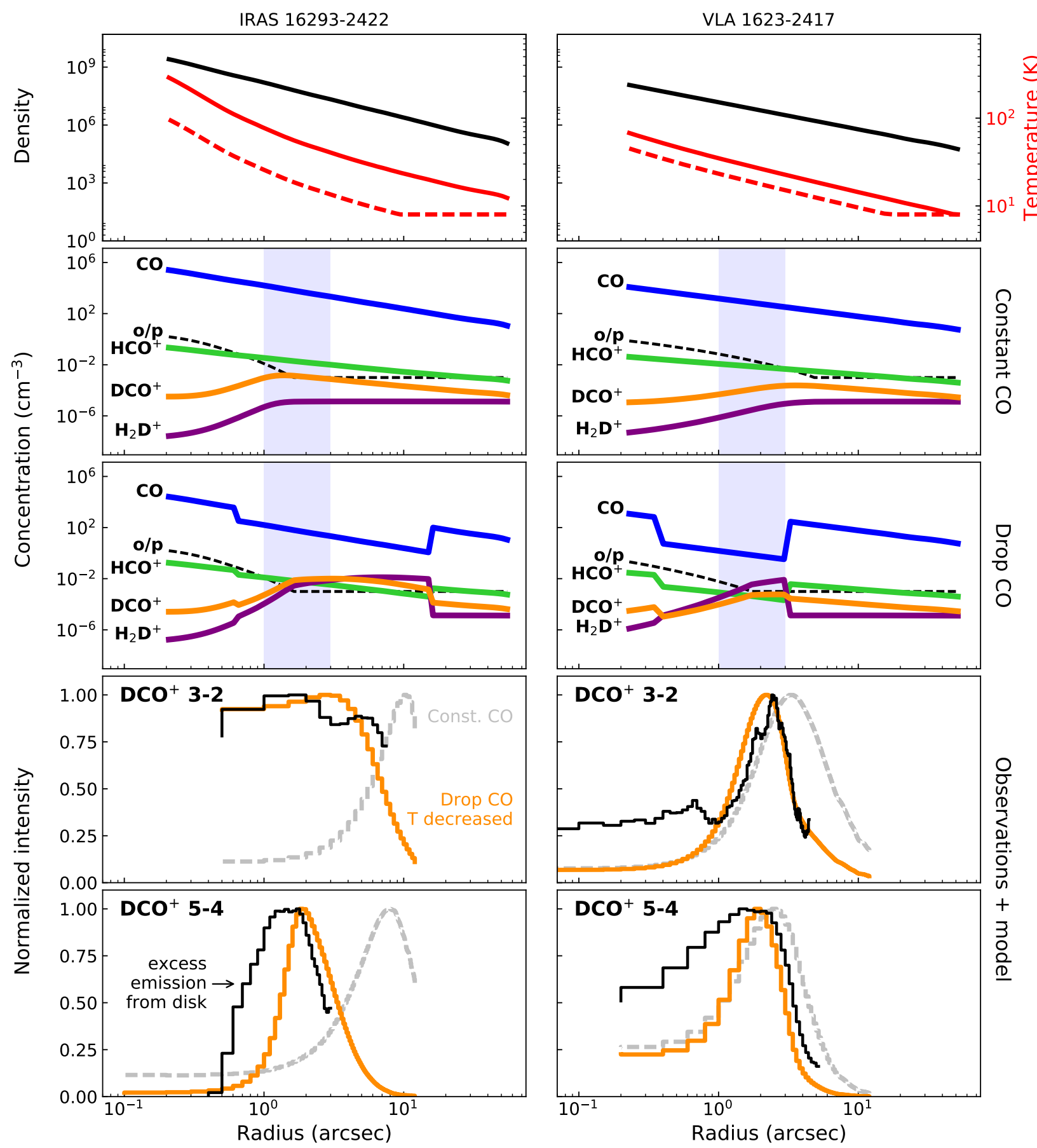

Fig. 8. Results from modeling the observed $\mathrm{DCO}^{+}$peak with our simple analytic chemical model. The left column shows results for IRAS 16293-2422, while the right column shows those for VLA 1623-2417. The top row shows the input density (black) and temperature (red) as functions of radius for each system. The solid red line shows the original temperature profile used for the constant CO model (second row). The dashed red line is the profile decreased by factors of 3 and 1.5 for IRAS 16293-2422 and VLA 1623-2417, respectively, and used for the drop CO model (third row). The lower limit on the temperature is set at $8 \mathrm{~K}$. In the second and third rows, the shaded range is the location of the observed $\mathrm{DCO}^{+}$peak. The fourth and fifth rows show the observed radial profiles of $\mathrm{DCO}^{+}$(black lines) overlaid with both Constant (dashed gray lines) and Drop CO models (solid orange lines). In all panels, the protostellar source is located on the left, and the envelope on the right, with the peak position of $\mathrm{DCO}^{+}$indicating the disk-envelope interface. We note that the excess $\mathrm{DCO}^{+} 5-4$ emission from the disk(-like) regions is not well reproduced by the cold $\mathrm{DCO}^{+}$network.

both IRAS 16293 and VLA 1623, the red-shifted peak emission is considered, since it is the most prominent. The results for IRAS 16293 and VLA 1623 are compared in Table 4.

For IRAS 16293, a ratio $\mathrm{DCO}^{+} 5-4 / 3-2=0.6 \pm 0.1$ is obtained from the PILS Band 7 observations and the SMA $230 \mathrm{GHz}$ observations (Jørgensen et al. 2011). We note that for
IRAS 16293, we only used the peak intensity from the ACA observations, and thus pick up emission from scales similar to the SMA observations. Thus, beam dilution does not need to be taken into consideration. We adopted a line width of $1.0 \mathrm{~km} \mathrm{~s}^{-1}$ and a column density of $3 \times 10^{12} \mathrm{~cm}^{-2}$, a value that also reproduces the observed line intensities. For densities 
Table 4. $\mathrm{DCO}^{+}$intensities and line ratios, with inferred temperature and density.

\begin{tabular}{|c|c|c|c|c|c|c|}
\hline Source & \multicolumn{2}{|c|}{ IRAS $16293-2422$} & \multicolumn{2}{|c|}{ VLA $1623-2417$ - ALMA } & \multicolumn{2}{|c|}{ VLA $1623-2417$ - APEX } \\
\hline Transition & 5-4 (ACA) & $3-2$ (SMA) & $5-4$ & $3-2$ & $5-4$ & $3-2$ \\
\hline Line width $\left(\mathrm{km} \mathrm{s}^{-1}\right)$ & 1.0 & 1.0 & 0.6 & 0.6 & 0.7 & 0.7 \\
\hline Beam $(\operatorname{arcsec})$ & $5.25 \times 2.36$ & $4.11 \times 2.45$ & $0.87 \times 0.65$ & $0.85 \times 0.54$ & 17.3 & 28.9 \\
\hline Peak $\left(\mathrm{Jy} \mathrm{beam}^{-1}\right)$ & $3.8 \pm 0.6$ & $1.9 \pm 0.2$ & $0.29 \pm 0.02$ & $0.087 \pm 0.008$ & & 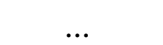 \\
\hline Peak $(\mathrm{K})$ & $2.9 \pm 0.4$ & $4.9 \pm 0.6$ & $4.8 \pm 0.3$ & $4.9 \pm 0.5$ & $0.79 \pm 0.1^{a}$ & $4.8 \pm 0.1$ \\
\hline Line ratio & \multirow{2}{*}{\multicolumn{2}{|c|}{$\begin{array}{l}0.6 \pm 0.1 \\
3 \times 10^{12}\end{array}$}} & \multirow{2}{*}{\multicolumn{2}{|c|}{$\begin{array}{l}1.0 \pm 0.1 \\
2 \times 10^{12}\end{array}$}} & \multirow{2}{*}{\multicolumn{2}{|c|}{$\begin{array}{c}0.2 \pm 0.03 \\
2 \times 10^{12}\end{array}$}} \\
\hline Column density $\left(\mathrm{cm}^{-2}\right)$ & & & & & & \\
\hline $\mathrm{H}_{2}$ density $\left(\mathrm{cm}^{-3}\right)$ & \multicolumn{2}{|c|}{$1-8 \times 10^{7}$} & \multirow{2}{*}{\multicolumn{2}{|c|}{$2-6 \times 10^{6}$}} & \multicolumn{2}{|c|}{$1-6 \times 10^{6}$} \\
\hline Kinetic temperature $(\mathrm{K})$ & \multicolumn{2}{|c|}{$20-23$} & & $30-55$ & \multicolumn{2}{|c|}{$12-19$} \\
\hline$\tau$ & \multicolumn{2}{|c|}{$<1$} & \multicolumn{2}{|c|}{$<1$} & \multicolumn{2}{|c|}{$<1$} \\
\hline
\end{tabular}

Notes. ${ }^{(a)}$ Peak temperature with beam dilution factor applied to $\mathrm{DCO}^{+} 5-4$ (see Appendix C), taking the beam to be $17.3^{\prime \prime}$, and source $28.9^{\prime \prime}$.
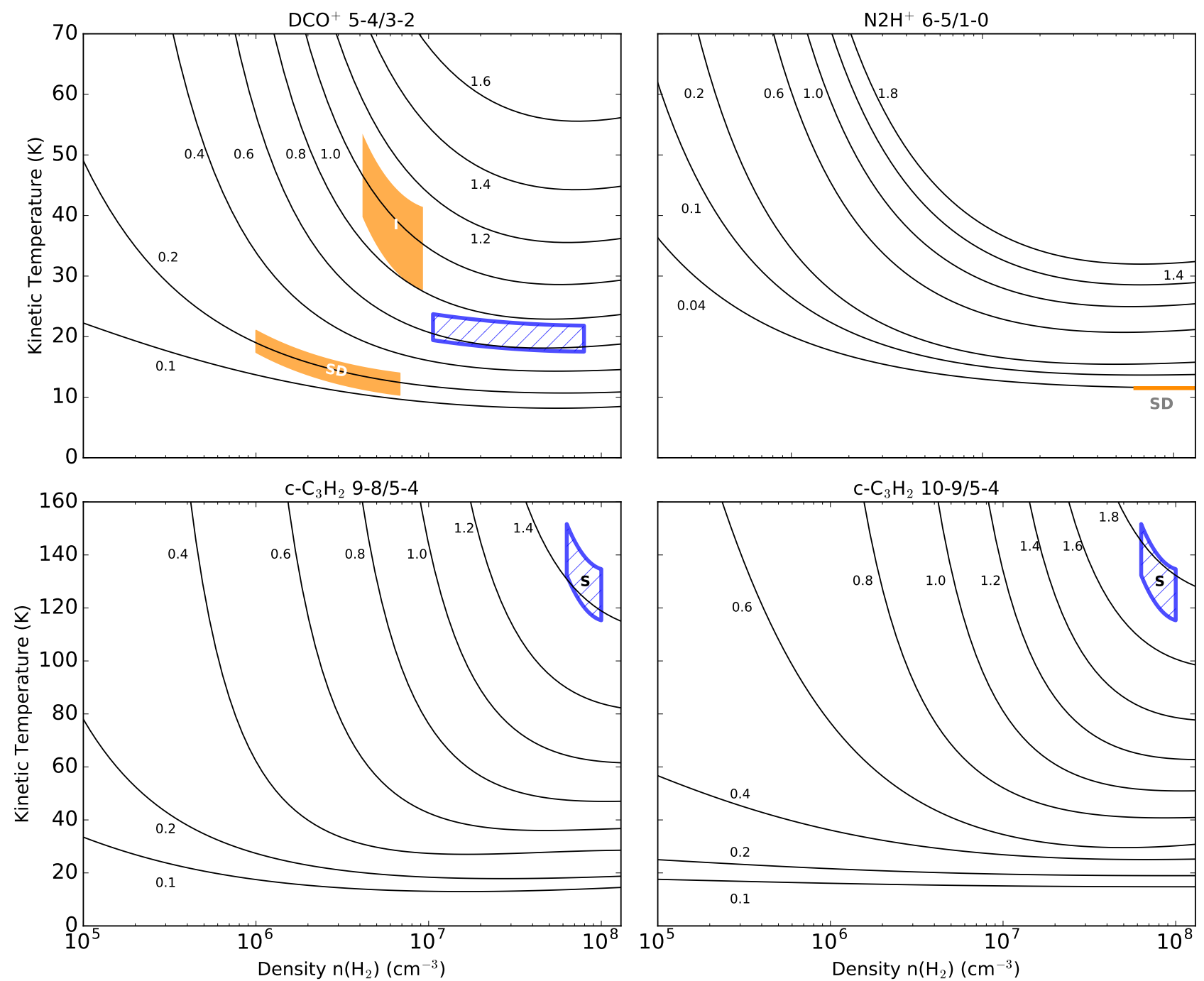

Fig. 9. Calculated line brightness temperature ratios for $\mathrm{DCO}^{+} 5-4 / 3-2, \mathrm{~N}_{2} \mathrm{H}^{+} 6-5 / 1-0$ and $\mathrm{c}-\mathrm{C}_{3} \mathrm{H}_{2} 10-9 / 5-4$, and 9-8/5-4. Black lines show the modeled ratios assuming column densities of $2.5 \times 10^{12}$, and $1.3 \times 10^{13} \mathrm{~cm}^{-2}$ for $\mathrm{DCO}^{+}$and $\mathrm{N}_{2} \mathrm{H}^{+}$, respectively, and $7 \times 10^{13}$ for both $\mathrm{c}-\mathrm{C}_{3} \mathrm{H}_{2}$ ratios. Colored regions indicate the observed line ratios drawn over the range of densities and temperatures that characterize the observed emission, for IRAS 16293-2422 with ALMA (hatched blue) and VLA 1623-2417 (solid orange) with single-dish (SD) and interferometric (I) observations. For $\mathrm{c}-\mathrm{C}_{3} \mathrm{H}_{2}$, the value for IRAS $16293-2422$ is from the south position.

below $10^{6} \mathrm{~cm}^{-3}$, the critical density of the 5-4 transition, the line ratio is primarily sensitive to density; at higher densities, the ratio becomes a good temperature probe. According to the density structure presented in Fig. 8 (top panel), the density at the 
Table 5. $\mathrm{c}-\mathrm{C}_{3} \mathrm{H}_{2}$ inferred parameters and abundance ratio for $\mathrm{c}-\mathrm{C}_{3} \mathrm{H}_{2} / \mathrm{C}_{2} \mathrm{H}$.

\begin{tabular}{|c|c|c|c|c|c|c|c|c|c|}
\hline \multirow[t]{2}{*}{ Position } & \multicolumn{2}{|c|}{ Coordinates } & \multirow[t]{2}{*}{$9-8 / 5-4$} & \multirow{2}{*}{$10-9 / 5-4$} & \multirow{2}{*}{$\begin{array}{c}n_{\mathrm{H}_{2}} \\
\left(\mathrm{~cm}^{-3}\right)\end{array}$} & \multirow{2}{*}{$\begin{array}{l}T_{\text {kin }} \\
(\mathrm{K})\end{array}$} & \multirow{2}{*}{$\begin{array}{l}N_{\mathrm{c}-\mathrm{C}_{3} \mathrm{H}_{2}}^{a, b} \\
\left(\mathrm{~cm}^{-2}\right)\end{array}$} & \multirow{2}{*}{$\begin{array}{l}N_{\mathrm{C}_{2} \mathrm{H}}^{a} \\
\left(\mathrm{~cm}^{-2}\right)\end{array}$} & \multirow[t]{2}{*}{$\mathrm{c}-\mathrm{C}_{3} \mathrm{H}_{2} / \mathrm{C}_{2} \mathrm{H}$} \\
\hline & RA & Dec & & & & & & & \\
\hline \multicolumn{10}{|c|}{ IRAS $16293-2422$} \\
\hline South & $16: 32: 22.88$ & $-24: 28: 39.78$ & $1.4 \pm 0.07$ & $1.7 \pm 0.08$ & $5-10 \times 10^{7}$ & $120-155$ & $9.3 \times 10^{13}$ & $\leq 3 \times 10^{13}$ & $\geq 3.1$ \\
\hline Center & $16: 32: 22.69$ & $-24: 28: 35.16$ & & & $1-6 \times 10^{7 c}$ & $50-120$ & $5-7 \times 10^{12}$ & $2 \times 10^{14}$ & $\leq 0.035$ \\
\hline North & $16: 32: 22.55$ & $-24: 28: 30.28$ & & & $1-6 \times 10^{7 c}$ & $50-120$ & $5-7 \times 10^{12}$ & $2 \times 10^{14}$ & $\leq 0.035$ \\
\hline \multicolumn{10}{|c|}{ VLA $1623-2417 \quad c-\mathrm{C}_{3} \mathrm{H}_{2} \quad 6_{0,6}-5_{1,5}$} \\
\hline $\mathrm{c}-\mathrm{C}_{3} \mathrm{H}_{2}{ }^{d}$ & $16: 26: 26.39$ & $-24: 24: 30.69$ & $\ldots$ & $\cdots$ & $5-10 \times 10^{7}$ & $120-155$ & $5.3-6.7 \times 10^{10}$ & $3 \times 10^{13}$ & $0.002^{e}$ \\
\hline
\end{tabular}

Notes. ${ }^{(a)}$ For IRAS $16293-2422$, column densities are for a beam of $0.5^{\prime \prime}$. For VLA 1623-2417, column densities are for a beam of $17.3^{\prime \prime}$, corresponding to the beam of the $\mathrm{C}_{2} \mathrm{H}$ observations. ${ }^{(b)} \mathrm{An} \mathrm{o} / \mathrm{p}=3$ was used to calculate the total column density of $\mathrm{c}-\mathrm{C}_{3} \mathrm{H}_{2} .{ }^{\left({ }^{(c)}\right.}$ Densities are assumed from the model envelope. ${ }^{(d)} \mathrm{c}-\mathrm{C}_{3} \mathrm{H}_{2} n_{\mathrm{H}_{2}}$ and $T_{\text {kin }}$ parameters taken from the south position of IRAS 16293-2422. ${ }^{(e)}$ Due to the different scales being picked up by the observations, the ratio is not well determined, and is provided here for reference.

peak $\mathrm{DCO}^{+}$emission position is higher than the critical density, so a kinetic temperature between 20 and $23 \mathrm{~K}$ can be inferred for IRAS 16293. This temperature is consistent with the chemical modeling of the $\mathrm{DCO}^{+}$peak position.

For VLA 1623, the ALMA $12 \mathrm{~m}$ array observations provide $\mathrm{DCO}^{+} 5-4 / 3-2=1.0 \pm 0.1$. The beam-size of $\mathrm{DCO}^{+}$ $5-4\left(0.87^{\prime \prime} \times 0.65^{\prime \prime}\right)$ is similar to that of the 3-2 transition $\left(0.87^{\prime \prime} \times 0.54^{\prime \prime}\right)$ and thus no beam dilution factor was added to the calculation. This line brightness temperature ratio implies a kinetic temperature between 30 and $55 \mathrm{~K}$, adopting a column density of $2 \times 10^{12}$ and a line width of $0.7 \mathrm{~km} \mathrm{~s}^{-1}$ to reproduce the observed peak intensities. This is higher than expected from the chemical modeling of $\mathrm{DCO}^{+}$. The APEX observations are used to double check if this is the kinetic temperature of the bulk of the $\mathrm{DCO}^{+}$emission at the disk-envelope interface. The APEX $\mathrm{DCO}^{+}$data give a much lower line ratio, 5-4/3-2 $=0.2 \pm 0.03$. This line brightness temperature ratio is well reproduced by a kinetic temperature of $12-19 \mathrm{~K}$, in agreement with the chemical model. It is likely that the ALMA $12 \mathrm{~m}$ array observations are picking up both warm and cold $\mathrm{DCO}^{+}$emission in the 5-4 transition, but only cold $\mathrm{DCO}^{+}$in the 3-2 transition. On the other hand, the APEX observations are recovering $\mathrm{DCO}^{+}$emission from the cold regions at the edge of the disk and the envelope, but the beam size dilutes the emission from the inner regions. This then causes the discrepancy of derived kinetic temperatures that we obtain from interferometric vs. singledish data. The temperature from the interferometric data is driven up due to more emission being detected in the higher transition.

\section{2. $\mathrm{C}-\mathrm{C}_{3} \mathrm{H}_{2}$ excitation}

Five transitions of $\mathrm{c}-\mathrm{C}_{3} \mathrm{H}_{2}$ are detected toward IRAS 16293. Temperature is derived from the $\mathrm{c}-\mathrm{C}_{3} \mathrm{H}_{2} 9-8 / 5-4$ and 10-9/5-4 ratios following the same method as in Sect. 4.1. The collisional rate coefficients for $\mathrm{c}-\mathrm{C}_{3} \mathrm{H}_{2}$ are based on Chandra \& Kegel (2000). The density range is chosen based on the envelope model of IRAS 16293. Figure 9 shows the line brightness temperature ratios as functions of $\mathrm{H}_{2}$ density and kinetic temperature. The ortho-c- $\mathrm{C}_{3} \mathrm{H}_{2}$ molecular file is used for the RADEX calculations since the 5-4 transition $(349.264 \mathrm{GHz})$ presented here is the ortho form (para-c- $\mathrm{C}_{3} \mathrm{H}_{2} 5-4$ is at $338.204 \mathrm{GHz}$ ). To convert to the total (ortho + para) $\mathrm{c}-\mathrm{C}_{3} \mathrm{H}_{2}$ column density, an $\mathrm{o} / \mathrm{p}$ ratio of 3 was used. Three regions covering the south $\mathrm{c}-\mathrm{C}_{3} \mathrm{H}_{2}$ peak and the $\mathrm{C}_{2} \mathrm{H}$ peaks near the center and north of the map are selected to derive the temperature and $\mathrm{c}-\mathrm{C}_{3} \mathrm{H}_{2} / \mathrm{C}_{2} \mathrm{H}$ column density ratios (Fig. 3). For the position with no detections of either molecule, the peak intensity from within the box used to probe the respective position (Fig. 3) is used. These values are listed in Table C.1. Table 5 lists the positions. The IRAS 16293 A and $\mathrm{B}$ positions are not modeled due to contamination from other molecular species.

Both $\mathrm{c}-\mathrm{C}_{3} \mathrm{H}_{2}$ 9-8/5-4 and 10-9/5-4 ratios are simultaneously fit for the south position. For the center and north positions, a temperature of $50-120 \mathrm{~K}$ is assumed and the column density is calculated assuming an upper limit of three times the rms noise for the peak temperature brightness. Table 5 lists the derived kinetic temperature from the $\mathrm{c}-\mathrm{C}_{3} \mathrm{H}_{2}$ ratio together with the assumed $\mathrm{c}-\mathrm{C}_{3} \mathrm{H}_{2}$ column densities for the regions being traced. The total column density for $\mathrm{c}-\mathrm{C}_{3} \mathrm{H}_{2}$ is calculated assuming an ortho-to-para ratio of 3 . The peak intensities for each transition are listed in Table C.1. We find that the temperature for the south $\mathrm{c}-\mathrm{C}_{3} \mathrm{H}_{2}$ peak, corresponding to the outflow cavity of IRAS $16293 \mathrm{~A}$, is between 120 and $155 \mathrm{~K}$. Comparing the temperatures obtained from $\mathrm{c}-\mathrm{C}_{3} \mathrm{H}_{2}$ and $\mathrm{DCO}^{+}$line ratios, it is clear that $\mathrm{c}-\mathrm{C}_{3} \mathrm{H}_{2}$ arises from a much warmer region than $\mathrm{DCO}^{+}$. This is linked to the spatial anti-correlation found for these two molecules, both in our observations and other work (see Sect. 5).

Only one transition of $\mathrm{c}-\mathrm{C}_{3} \mathrm{H}_{2}$ is available for VLA 1623 , with an upper level energy (38.6 K) lower than those observed toward IRAS 16293 ( $\geq 49 \mathrm{~K}$ ). Thus, to obtain an idea of the column densities in the region traced by $\mathrm{c}-\mathrm{C}_{3} \mathrm{H}_{2}$ toward VLA 1623 , we adopt the temperature and density from the $\mathrm{c}-\mathrm{C}_{3} \mathrm{H}_{2}$ line ratios toward IRAS 16293 at the south peak. The $\mathrm{c}-\mathrm{C}_{3} \mathrm{H}_{2}$ south peak of IRAS 16293 is chosen due to the fact that it traces the outflow cavity, as it does for VLA 1623. Using the parameters of $\mathrm{c}-\mathrm{C}_{3} \mathrm{H}_{2}$ from the south position of IRAS 16293, the derived column density is $2 \times 10^{13} \mathrm{~cm}^{-2}$ for a beam of $1.6^{\prime \prime} \times 0.88^{\prime \prime}$, lower than that found for the south peak of IRAS 16293 by about a factor of 5, and similar to the column density found for the central position of the IRAS 16293 map. If the beam from the $\mathrm{C}_{2} \mathrm{H}$ observations is considered $\left(17.3^{\prime \prime}\right)$, the derived column density of $\mathrm{c}-\mathrm{C}_{3} \mathrm{H}_{2}$ becomes a few $10^{10} \mathrm{~cm}^{-2}$ (Table 5).

\section{3. $\mathrm{c}-\mathrm{C}_{3} \mathrm{H}_{2} / \mathrm{C}_{2} \mathrm{H}$ abundance ratio}

The same transitions of $\mathrm{C}_{2} \mathrm{H}$ are observed toward both sources, with ALMA for IRAS 16293 and with APEX for VLA 1623. The peak intensities are listed in Table C.1. Since $\mathrm{C}_{2} \mathrm{H}$ ratios 
are not sensitive to temperature or density given the similar upper eneriges $E_{\text {up }}$ (Table 1), the method adopted for $\mathrm{DCO}^{+}$and $\mathrm{c}-\mathrm{C}_{3} \mathrm{H}_{2}$ cannot be used here. Instead, the $\mathrm{C}_{2} \mathrm{H}$ column density is derived by assuming the kinetic temperature and number density obtained from $\mathrm{c}-\mathrm{C}_{3} \mathrm{H}_{2}$ line ratios. Using the same derived temperature and density for $\mathrm{C}_{2} \mathrm{H}$ from $\mathrm{c}-\mathrm{C}_{3} \mathrm{H}_{2}$ for the corresponding position is done to probe the parameters of $\mathrm{C}_{2} \mathrm{H}$ if it is tracing the same region as $\mathrm{c}-\mathrm{C}_{3} \mathrm{H}_{2}$. The results are listed in Table 5 .

The south position in IRAS 16293 presents a column density of $\leq 3 \times 10^{13} \mathrm{~cm}^{-2}$ for $\mathrm{C}_{2} \mathrm{H}$, about an order of magnitude lower compared to the center and north positions which have a column density of $2 \times 10^{14} \mathrm{~cm}^{-2}$. Table 5 lists the $\mathrm{c}-\mathrm{C}_{3} \mathrm{H}_{2} / \mathrm{C}_{2} \mathrm{H}$ column density ratio for each position. It must be noted that the ratio at the south position is a lower limit, whereas for the central and north position, it is an upper limit. The differences in ratios between positions reflect the anti-correlation of the two molecules in the IRAS 16293 system. Most certainly, the anti-correlation is not due to critical densities, since the derived number densities of $\mathrm{c}-\mathrm{C}_{3} \mathrm{H}_{2}\left(10^{7}\right.$ to $\left.10^{8} \mathrm{~cm}^{-3}\right)$ at all points are above the critical densities of both $\mathrm{C}_{2} \mathrm{H}\left(8 \times 10^{4}\right.$ to $\left.6 \times 10^{5} \mathrm{~cm}^{-3}\right)$ and $\mathrm{c}-\mathrm{C}_{3} \mathrm{H}_{2}\left(2-5 \times 10^{5} \mathrm{~cm}^{-3}\right)$.

For VLA 1623, $\mathrm{C}_{2} \mathrm{H}$ column densities are found to be a few times $10^{13} \mathrm{~cm}^{-2}$, lower than the peaks of $\mathrm{C}_{2} \mathrm{H}$ detected toward IRAS 16293. The difference in column densities are most likely be due to beam dilution effect from the observations with APEX. Thus the $\mathrm{c}-\mathrm{C}_{3} \mathrm{H}_{2} / \mathrm{C}_{2} \mathrm{H}$ ratio is not well determined for VLA1623. The results are listed in Table 5.

\section{4. $\mathrm{N}_{2} \mathrm{H}^{+}$and $\mathrm{N}_{2} D^{+}$}

For VLA 1623, the ALMA $12 \mathrm{~m}$ array observations of $\mathrm{N}_{2} \mathrm{D}^{+}$ and $\mathrm{N}_{2} \mathrm{H}^{+}$did not detect any emission. Since these molecules are readily detected in other sources (e.g., Tobin et al. 2013), the cause of this nondetection is examined. Two cases are explored, extended and compact emission concentrated in a $1^{\prime \prime}$ region. The details of the analysis are given in Appendix C.

In the case of extended emission, the predicted $\mathrm{N}_{2} \mathrm{H}^{+} 4-3$ peak intensity is the same as the noise level of our observations, while for the case of the emission concentrated in 1 " region, the $S / N$ would be about 26 . In a similar manner, the predicted $\mathrm{N}_{2} \mathrm{D}^{+} 3-2$ peak intensity is expected to have a marginal detection in our observations for the extended emission case, and a $S / N=$ 40 for the compact emission case. Thus, we should have detected both molecules in our observations if they arose from a compact structure.

\section{Discussion}

\subsection{Comparison of IRAS 16293-2422 and VLA 1623-2417}

The chemical structure of both systems is compared in this section. A cartoon of their structure is given in Fig. 10.

The $\mathrm{DCO}^{+}$peak position in both sources is well described by a drop in the temperature profile. This drop can be explained by the presence of a disk which shadows the envelope, causing the $\mathrm{DCO}^{+}$emission to move inward, closer to the source along the disk plane. For VLA 1623, the ALMA DCO ${ }^{+} 5-4$ observations are picking up emission coming from both the cold envelope at the edge of the disk (disk-envelope interface) and the warmer parts of the disk.

The outflow cavity wall of IRAS 16293 A and VLA 1623 A is traced by $\mathrm{c}-\mathrm{C}_{3} \mathrm{H}_{2}$. Due to $\mathrm{c}-\mathrm{C}_{3} \mathrm{H}_{2}$ having both top-down and bottom up chemistry, it was not studied using a chemical

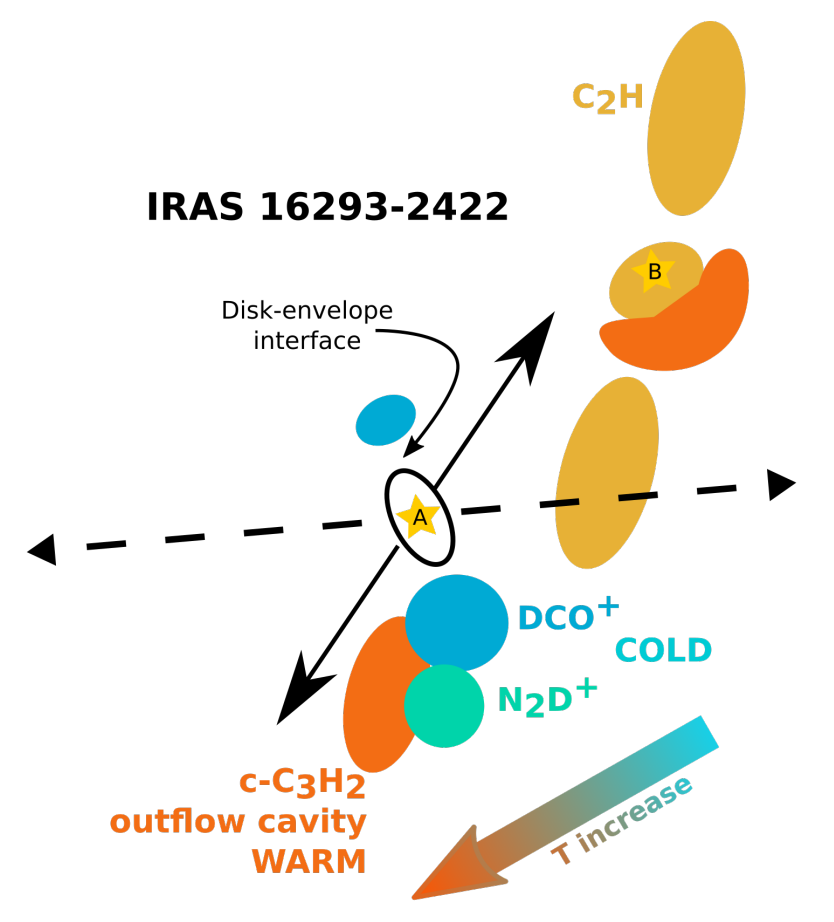

VLA 1623-2417

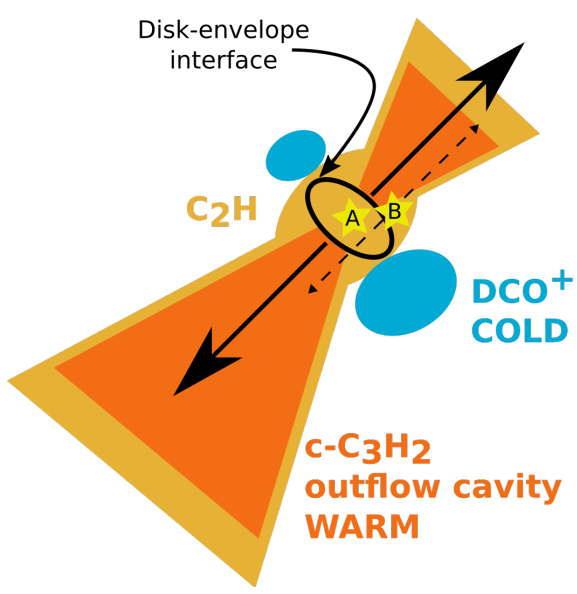

Fig. 10. Cartoon showing distribution of the molecules studied in this work toward both sources. The solid and dashed lines show outflow directions. Black ellipses indicate disk structures. $\mathrm{C}_{2} \mathrm{H}$ toward VLA 1623-2417 A is observed with APEX, thus the distribution is expected based on the physical conditions of VLA 1623-2417, instead of direct mapping.

model. For VLA $1623 \mathrm{~A}, \mathrm{c}-\mathrm{C}_{3} \mathrm{H}_{2}$ traces the full outflow cavity (extending out to $3^{\prime \prime}$ ), whereas for IRAS 16293 A only one side of the south outflow cavity wall is observed. It is possible that the $\mathrm{c}-\mathrm{C}_{3} \mathrm{H}_{2}$ emission is product of UV radiation from the central source, and the warmer temperatures found in the outflow cavity. UV radiation liberates atomic carbon which leads to gas-phase formation of small hydrocarbons. Chemical models of the outflow cavity walls show that $\mathrm{c}-\mathrm{C}_{3} \mathrm{H}_{2}$ is initially concentrated around the outflow wall, and progressively moves to the disk plane as the cavity widens with age, irradiating more envelope material (Drozdovskaya et al. 2015). Higher temperatures could accelerate certain chemical processes in the protostellar envelope, while movement of material through outflows, rotation, and infall could bring dust with $\mathrm{c}-\mathrm{C}_{3} \mathrm{H}_{2}$ precursors (e.g., $\mathrm{CH}_{4}, \mathrm{C}_{2} \mathrm{H}_{2}$ ) closer to areas where they can be sublimated 
and thus enhance the $\mathrm{c}-\mathrm{C}_{3} \mathrm{H}_{2}$ gas. Hence, the differences in the spatial distributions of $\mathrm{c}-\mathrm{C}_{3} \mathrm{H}_{2}$ in IRAS $16293 \mathrm{~A}\left(L_{\mathrm{bol}}>18 L_{\odot}\right.$; Jacobsen et al. 2018) and VLA $1623 \mathrm{~A}\left(L_{\mathrm{bol}} \sim 1 L_{\odot}\right)$ could be the product of age, luminosity, or core dynamics. In any case, the presence of $\mathrm{c}-\mathrm{C}_{3} \mathrm{H}_{2}$ emission is due to the temperature of the region where it is observed.

The molecule $\mathrm{C}_{2} \mathrm{H}$ does not present similar distributions in IRAS 16293 and VLA 1623. For VLA 1623, the single-dish observations of $\mathrm{C}_{2} \mathrm{H}$ showed no emission was detected in the off positions, thus we expect that $\mathrm{C}_{2} \mathrm{H}$ peaks around VLA $1623 \mathrm{~A}$. Given the observations, low temperatures in the envelope of VLA $1623 \mathrm{~A}$ as well as the envelopes of VLA $1623 \mathrm{~B}$ and W, and the formation mechanism of the two molecules, it might be possible that $\mathrm{C}_{2} \mathrm{H}$ is spatially correlated with $\mathrm{c}-\mathrm{C}_{3} \mathrm{H}_{2}$ on $\sim 15^{\prime \prime}$ scales. However, interferometric observations are needed to verify if both molecules are correlated toward VLA 1623. On larger scales, part of the $\mathrm{C}_{2} \mathrm{H}$ could also come from the envelope, given that it shows similar broadening with $\mathrm{DCO}^{+}$(Lindberg et al. 2017).

For IRAS 16293, the observed $\mathrm{C}_{2} \mathrm{H}$ is found in the region one beam away from IRAS $16293 \mathrm{~B}$, but does not show relation with the position or outflow of IRAS 16293 A, nor with the dust ridge connecting both sources. Beyond IRAS $16293 \mathrm{~B}$, $\mathrm{C}_{2} \mathrm{H}$ and $\mathrm{c}-\mathrm{C}_{3} \mathrm{H}_{2}$ are not spatially correlated (Figs. 3 and B.1). This anti-correlation is unexpected from chemical models (e.g., Gerin et al. 2011; Drozdovskaya et al. 2015; Guzmán et al. 2015) or observations (see Sect. 5.2). The $\mathrm{c}-\mathrm{C}_{3} \mathrm{H}_{2} / \mathrm{C}_{2} \mathrm{H}$ ratio is expected to be lowered with age, that is as the protostar evolves (O. Sipilä, priv. comm.). If the different distributions of $\mathrm{c}-\mathrm{C}_{3} \mathrm{H}_{2}$ and $\mathrm{C}_{2} \mathrm{H}$ are product of age, it would suggest that IRAS 16293 and VLA 1623 are young; however, lowering the ratio with age does not explain the anti-correlation observed within IRAS 16293.

A possible explanation may be top-down chemistry producing small hydrocarbons through UV destruction of large aromatic molecules as inferred for lower density PDRs (Guzmán et al. 2015), producing different amounts of the two molecules. Another possibility for the anti-correlation might be explained by the destruction of $\mathrm{C}_{2} \mathrm{H}$ in reactions with sulfur, nitrogen, oxygen or carbon chains (Sakai \& Yamamoto 2013). The full Band 7 spectrum is examined at the $\mathrm{c}-\mathrm{C}_{3} \mathrm{H}_{2}$ peak position (Figs. D.1-D.3) in order to examine whether products of $\mathrm{C}_{2} \mathrm{H}$ reactions are present. $\mathrm{C}_{2} \mathrm{~S}$, product of $\mathrm{C}_{2} \mathrm{H}$ reacting with sulfur (Drozdovskaya et al. 2018), is not detected. In fact, little else is observed in the dense gas south of IRAS 16293 A. Apart from c- $\mathrm{C}_{3} \mathrm{H}_{2}$, only $\mathrm{H}_{2} \mathrm{CS}$ (Drozdovskaya et al. 2018) and a few common species like $\mathrm{HCO}^{+}, \mathrm{H}_{2} \mathrm{CO}$; and $\mathrm{CH}_{3} \mathrm{OH}$ are detected, which is unexpected given that outflow cavity would be irradiated and encourage chemical complexity (Drozdovskaya et al. 2015). Reactions of $\mathrm{C}_{2} \mathrm{H}$ with carbon chains $\mathrm{C}_{n}$ would cause production of $\mathrm{C}_{n+2}$ and hydrogen, rendering these products undetectable due to lack of dipole moments. Overall, the strong anti-correlation of these molecules remains a chemical puzzle.

We detect $\mathrm{N}_{2} \mathrm{D}^{+} 7^{\prime \prime}$ south of IRAS 16293 A with the SMA (Fig. 4), bordering the $\mathrm{DCO}^{+}$emission (Jørgensen et al. 2011). In contrast, $\mathrm{N}_{2} \mathrm{D}^{+}$and $\mathrm{N}_{2} \mathrm{H}^{+}$are not detected with ALMA observations toward VLA 1623. Single-dish observations show an offset of about $60^{\prime \prime}$ between the position of VLA 1623 and the peak of $\mathrm{N}_{2} \mathrm{H}^{+}$and $\mathrm{N}_{2} \mathrm{D}^{+}$(Di Francesco et al. 2004; André et al. 2007; Liseau et al. 2015; Punanova et al. 2016; Favre et al. 2017). Line ratios of $\mathrm{N}_{2} \mathrm{H}^{+}$and $\mathrm{N}_{2} \mathrm{D}^{+}$from single-dish observations were investigated in Sect. 4.4 and Appendix C, where it was found that ALMA would detect only compact emission. Given the lack of ALMA detection of both molecules, $\mathrm{N}_{2} \mathrm{D}^{+}$and $\mathrm{N}_{2} \mathrm{H}^{+}$trace extended emission outside the envelope of VLA 1623 (Liseau et al. 2015).

The presence of $\mathrm{N}_{2} \mathrm{D}^{+}$in IRAS 16293, but not in VLA 1623, could be product of temperature differences. $\mathrm{N}_{2}$ can be frozen out onto dust grains at temperatures below $20 \mathrm{~K}$ (Bisschop et al. 2006), a scenario also pointed out by Di Francesco et al. (2004). While other nitrogen-bearing molecules such as $\mathrm{CN}$, $\mathrm{HCN}, \mathrm{HNC}$, and NO can form in the gas and on grain surfaces, $\mathrm{N}_{2} \mathrm{H}^{+}$and $\mathrm{N}_{2} \mathrm{D}^{+}$only form in the gas phase if $\mathrm{N}_{2}$ gas is present. This scenario is further supported by the low temperatures found for $\mathrm{DCO}^{+}$. For VLA $1623 \mathrm{~A}, \mathrm{DCO}^{+}$has $T_{\text {kin }}=17 \mathrm{~K}$, and the chemical modeling suggests dust temperatures between 11 and $16 \mathrm{~K}$ for where $\mathrm{DCO}^{+}$peaks. This would indicate that further out, the temperature is even lower. In addition, at densities below $\sim 10^{4} \mathrm{~cm}^{-3}$ (two orders of magnitude below what is derived from $\mathrm{DCO}^{+}$for VLA $1623 \mathrm{~A}$ ), the dust and gas temperatures decouple, and without any additional external pressure, the gas temperature drops down to $10 \mathrm{~K}$ (Galli et al. 2002; Evans et al. 2001), which could cause $\mathrm{N}_{2} \mathrm{H}^{+}$and $\mathrm{N}_{2} \mathrm{D}^{+}$to recombine onto the dust grains or the precursor $\mathrm{N}_{2}$ to freeze-out. In contrast, $\mathrm{DCO}^{+}$south of IRAS $16293 \mathrm{~A}$ indicates $T_{\text {kin }}=20-23 \mathrm{~K}$ for the gas, and dust temperatures between 17 and $19 \mathrm{~K}$ from chemical modeling, evidencing that the envelope of IRAS $16293 \mathrm{~A}$ is warmer than that of VLA 1623 A (Jacobsen et al. 2018).

The molecules $\mathrm{N}_{2} \mathrm{H}^{+}$and $\mathrm{N}_{2} \mathrm{D}^{+}$are thought to be tracers of evolutionary stage (Emprechtinger et al. 2009), as well as of the CO snowline (Jørgensen et al. 2004; Anderl et al. 2016; van't Hoff et al. 2017). These assumptions break down for very cold envelopes of embedded protostars, like that of VLA 1623. Given that some starless cores do show $\mathrm{N}_{2} \mathrm{H}^{+}$and $\mathrm{N}_{2} \mathrm{D}^{+}$(e.g., Crapsi et al. 2005; Tobin et al. 2013), including the starless cores north of VLA 1623 (Di Francesco et al. 2004; Friesen et al. 2014), it cannot be said that the cold envelope itself is an indicator of evolutionary stage. It may be possible that the ridge of material north of VLA 1623, which contains the starless cores, is being heated somehow from the side, but VLA 1623 is being shielded and thus much colder (Di Francesco et al. 2004; Bergman et al. 2011; Friesen et al. 2014). VLA 1623 A itself is certainly heating up the disk and outflow cavity, evidenced by $\mathrm{DCO}^{+} 5-4$ emission on the disk and the presence of $\mathrm{c}-\mathrm{C}_{3} \mathrm{H}_{2}$ and $\mathrm{C}_{2} \mathrm{H}$, but on much smaller scales $(<100 \mathrm{AU})$ than in IRAS 16293 because of its lower luminosity.

\subsection{Comparison with starless cores and low-mass protostars}

In this section, IRAS 16293 and VLA 1623 are placed in the big picture of star formation. For this reason, the two systems described in the previous section are compared with observations of starless cores, embedded low-mass protostars and disks found in the literature. In addition, the multiplicity of the systems is also taken into consideration.

The starless core $\mathrm{L} 1544$ exhibits $\mathrm{c}-\mathrm{C}_{3} \mathrm{H}_{2}$ close to the dense cloud core center and away from cold regions traced by $\mathrm{DCO}^{+}$ (Spezzano et al. 2016a,b). This points to an anti-correlation between the chemistry traced by $\mathrm{DCO}^{+}$and that by $\mathrm{c}-\mathrm{C}_{3} \mathrm{H}_{2}$, which is present in both IRAS 16293 and VLA 1623. In the system NGC 1333 IRAS $4, \mathrm{C}_{2} \mathrm{H}$ is observed to peak on-source toward each component, including the starless core IRAS 4C, which has the strongest emission (Koumpia et al. 2016, 2017). In the young embedded object IRAS $15398, \mathrm{C}_{2} \mathrm{H}$ traces the redand blue-shifted outflow cavity (Jørgensen et al. 2013). In contrast, L1527 presents both $\mathrm{C}_{2} \mathrm{H}$ and $\mathrm{c}-\mathrm{C}_{3} \mathrm{H}_{2}$ in the envelope and disk component, with enhancements at the centrifugal barrier (Sakai et al. 2010, 2014a, 2016), but no emission along the 
outflow cavity. The spatial distribution of $\mathrm{C}_{2} \mathrm{H}$ and $\mathrm{c}-\mathrm{C}_{3} \mathrm{H}_{2}$ is similar in L1527, with the emission from $\mathrm{c}-\mathrm{C}_{3} \mathrm{H}_{2}$ being more compact than that of $\mathrm{C}_{2} \mathrm{H}$. In Oph-IRS67, $\mathrm{C}_{2} \mathrm{H}$ and $\mathrm{c}-\mathrm{C}_{3} \mathrm{H}_{2}$ exist in the same region, although the spatial extent is not the same (Artur de la Villarmois et al. 2018). In the protoplanetary disk TW Hya, $\mathrm{C}_{2} \mathrm{H}$ and $\mathrm{c}-\mathrm{C}_{3} \mathrm{H}_{2}$ are found to reside in the disk, bordering the millimeter dust, with both molecules showing an identical spatial distribution (Bergin et al. 2016). The $\mathrm{c}-\mathrm{C}_{3} \mathrm{H}_{2}$ and $\mathrm{C}_{2} \mathrm{H}$ distribution toward VLA 1623 is consistent with that observed in other protostellar systems; however, for IRAS 16293 the lack of correlation between the two molecules is still a puzzle, since no other low-mass protostar or starless core reported in the literature at present presents this situation.

In NGC1333 SVS13, $\mathrm{N}_{2} \mathrm{H}^{+}$is detected around two of the four components of the system (Chen et al. 2009). From the system, SVS13B and SVS13C are Class 0 protostars, but the first has $\mathrm{N}_{2} \mathrm{H}^{+}$emission while the second does not. Thus, the uneven distribution of material is not related to the evolutionary stage, but instead is most likely related to the varying envelope temperature.

Several of the systems mentioned above are multiple protostars, as are IRAS 16293 and VLA 1623. The chemical structure is found to not be homogeneous among the individual components of these systems. This would suggest that the components of wide multiple protostellar systems have no effect on the chemistry of each other. For close multiple protostellar systems, the only case shown here is that of VLA $1623 \mathrm{~A}$ and B, which have a separation of $\sim 200 \mathrm{AU}$ (based on the disk radius of VLA $1623 \mathrm{~A}$ and a lack of disturbance of the disk by VLA 1623 B) and show different chemical structures. However, these are only two cases, and more observations of multiple protostellar systems are needed to further understand the effect of companions on chemical structure.

\subsection{Comparison with diffuse clouds, PDRs, and intermediate to high-mass protostars}

Looking to compare what structures are common throughout the interstellar medium, we compare the distributions found in IRAS 16293 and VLA 1623 with diffuse clouds and PDRs. Furthermore, given that IRAS 16293 is much warmer than VLA 1623, it is also compared to high-mass protostars.

Toward the Horsehead nebula PDR, $\mathrm{DCO}^{+}$is observed far from the irradiated edge of the region, with no emission at the edge (Guzmán et al. 2015). The spatial anti-correlation between $\mathrm{DCO}^{+}$and $\mathrm{c}-\mathrm{C}_{3} \mathrm{H}_{2}$ or $\mathrm{C}_{2} \mathrm{H}$ suggests a temperature effect, as found for IRAS 16293 and VLA 1623, highlighting that $\mathrm{DCO}^{+}$ is a really good tracer of cold regions.

Close correlation in spatial distribution $\mathrm{c}-\mathrm{C}_{3} \mathrm{H}_{2}$ and $\mathrm{C}_{2} \mathrm{H}$ is shown toward a number of PDRs, including the Orion Bar (Pety et al. 2007; van der Wiel et al. 2009; Nagy et al. 2015) and the Horsehead Nebula (Cuadrado et al. 2015; Guzmán et al. 2015), with both molecular species sitting at the irradiated, and thus warmer, edge of the region. In addition, a tight correlation between $\mathrm{c}-\mathrm{C}_{3} \mathrm{H}_{2}$ and $\mathrm{C}_{2} \mathrm{H}$ in diffuse clouds has been found (Lucas \& Liszt 2000; Gerin et al. 2011; Liszt et al. 2012). The column density $\mathrm{c}-\mathrm{C}_{3} \mathrm{H}_{2} / \mathrm{C}_{2} \mathrm{H}$ ratios calculated toward IRAS 16293 in the center and north positions $\left(\mathrm{c}-\mathrm{C}_{3} \mathrm{H}_{2} / \mathrm{C}_{2} \mathrm{H} \leq 0.035\right)$ reflect the values found for diffuse clouds $\left(\mathrm{c}-\mathrm{C}_{3} \mathrm{H}_{2} / \mathrm{C}_{2} \mathrm{H}=0.048\right.$; Lucas \& Liszt 2000; Liszt et al. 2012) and the envelope of $\mathrm{L} 1527\left(\mathrm{c}-\mathrm{C}_{3} \mathrm{H}_{2} / \mathrm{C}_{2} \mathrm{H}=0.035-0.06\right.$; Sakai et al. 2014a).

In high-mass star-forming regions, $\mathrm{c}-\mathrm{C}_{3} \mathrm{H}_{2}$ and $\mathrm{C}_{2} \mathrm{H}$ tend to be strongly correlated, with both lines presenting similar spatial distributions (Mookerjea et al. 2012, 2014; Pilleri et al. 2013). In contrast, toward IRAS $20343+4129, \mathrm{c}-\mathrm{C}_{3} \mathrm{H}_{2}$ and $\mathrm{C}_{2} \mathrm{H}$ show an anti-correlation around the outflow cavity walls of IRS 1 (Fontani et al. 2012) but not around the UC HII-region of IRS 3. The anti-correlation is explained to possibly be product of the gas density, with an enhancement of $\mathrm{C}_{2} \mathrm{H}$ located in the regions with denser gas. Thus, the warmer envelope of IRAS 16293 does not provide a solution to the puzzle of why $\mathrm{c}-\mathrm{C}_{3} \mathrm{H}_{2}$ and $\mathrm{C}_{2} \mathrm{H}$ are anti-correlated in this system, but the difference in density, possibly caused by the outflow direction shift, might provide a possible explanation for the anti-correlation of the two molecules. Chemical processes could also be playng a role in the anti-correlation of $\mathrm{c}-\mathrm{C}_{3} \mathrm{H}_{2}$ and $\mathrm{C}_{2} \mathrm{H}$ toward IRAS 16293.

\section{Conclusions}

In this work, we present ALMA, SMA, and APEX observations of $\mathrm{DCO}^{+}, \mathrm{c}-\mathrm{C}_{3} \mathrm{H}_{2}, \mathrm{C}_{2} \mathrm{H}, \mathrm{N}_{2} \mathrm{H}^{+}$, and $\mathrm{N}_{2} \mathrm{D}^{+}$toward IRAS 16293-2422 and VLA 1623-2417, both multiple protostellar systems in $\rho$ Ophiuchus. The spatial distribution of each molecule is compared for both systems. $\mathrm{DCO}^{+}$is studied using a simple analytic chemical network coupled with radiative transfer modeling, detailed in Murillo et al. (2015), in order to determine the conditions leading to the observed peak position. Non-LTE molecular excitation and radiative transfer modeling of the observed line brightness temperature ratios is done to derive physical parameters of the regions being traced by the molecules. Finally, the observations and results of VLA 1623-2417 and IRAS 16293-2422 are compared, both between the two sources and other objects, ranging from low- to high-mass protostars, diffuse clouds and PDRs, in order to understand what structures are common.

From this work, we extract the following key points:

1. Temperature is a controlling factor of the chemical structure of a protostellar system. Disks can alter the temperature of the envelope, while UV heating can encourage the start of chemical processes in the outflow cavity.

2. An asymmetric $\mathrm{DCO}^{+}$structure is a good tell-tale sign for the presence of a disk, since a disk shadows the envelope at its edge, lowering the temperature and causing $\mathrm{DCO}^{+}$to move inward only along the disk plane.

3. $\mathrm{c}-\mathrm{C}_{3} \mathrm{H}_{2}$ traces the outflow cavity of IRAS $16293-2422$ and VLA 1623-2417, but shows no disk component for either source.

4. Despite both VLA 1623-2417 and IRAS 16293-2422 being low-mass Class 0 embedded objects, their structure and chemical richness varies considerably, with VLA 1623-2417 being line poor. Its much lower luminosity, and consequently lower temperatures, coupled with a large cold disk, are likely to be at the root of this difference.

Although only two sources have been studied in this work and some results in the literature, there is evidence pointing to a lack of correlation between multiplicity and the chemical structure of the envelope of these systems, at least in the embedded phase. Nevertheless, multiple systems do provide an interesting way to compare the structure with similar conditions. It would be possible, however, that the heating from companion sources would affect the chemistry as the envelope clears. Further comparison of embedded multiple protostellar systems is needed to confirm these results.

Acknowledgements. The authors thank O. Sipilä, P. Caselli, S. Spezzano, N. Sakai, C. Favre, and N. Ligterink for helpful discussions on chemistry. This paper made use of the following ALMA data: ADS/JAO.ALMA 2011.0.00902.S, 
2013.1.01004.S, and 2013.1.00278.S. ALMA is a partnership of ESO (representing its member states), NSF (USA), and NINS (Japan), together with NRC (Canada) and NSC and ASIAA (Taiwan), in cooperation with the Republic of Chile. The Joint ALMA Observatory, is operated by ESO, AUI/NRAO, and NAOJ. This publication is partially based on data acquired with the Atacama Pathfinder Experiment (APEX). APEX is a collaboration between the Max-Planck-Institut fur Radioastronomie, the European Southern Observatory, and the Onsala Space Observatory. We are grateful to the APEX staff for support with the observations of VLA 1623-2417. Astrochemistry in Leiden is supported by the European Union A-ERC grant 291141 CHEMPLAN, by the Netherlands Research School for Astronomy (NOVA), by a Royal Netherlands Academy of Arts and Sciences (KNAW) professor prize. The group of J.K.J. acknowledges support from the European Research Council (ERC) under the European Union's Horizon 2020 research and innovation programme (grant agreement No 646908) through ERC Consolidator Grant "S4F". Research at Centre for Star and Planet Formation is funded by the Danish National Research Foundation. M.N.D. acknowledges the financial support of the Center for Space and Habitability (CSH) Fellowship and the IAU Gruber Foundation Fellowship.

\section{References}

Aikawa, Y., \& Herbst, E. 1999, A\&A, 351, 233

Aikawa, Y., Umebayashi, T., Nakano, T., \& Miyama, S. M. 1999, ApJ, 519, 705

Aikawa, Y., Wakelam, V., Hersant, F., Garrod, R. T., \& Herbst, E. 2012, ApJ, 760, 40

Anderl, S., Maret, S., Cabrit, S., et al. 2016, A\&A, 591, A3

André, P., Martin-Pintado, J., Despois, D., \& Montmerle, T. 1990, A\&A, 236, 180

André, P., Belloche, A., Motte, F., \& Peretto, N. 2007, A\&A, 472, 519

Artur de la Villarmois, E., Kristensen, L. E., Jørgensen, J. K., et al. 2018, A\&A, 614, A26

Bacmann, A., Taquet, V., Faure, A., Kahane, C., \& Ceccarelli, C. 2012, A\&A 541, L12

Belitsky, V., Lapkin, I., Monje, R., et al. 2006, in Proc. SPIE, SPIE Conf. Ser., $6275,62750 \mathrm{G}$

Bergin, E. A., Du, F., Cleeves, L. I., et al. 2016, ApJ, 831, 101

Bergman, P., Parise, B., Liseau, R., \& Larsson, B. 2011, A\&A, 527, A39

Bergner, J. B., Öberg, K. I., Garrod, R. T., \& Graninger, D. M. 2017, ApJ, 841, 120

Bisschop, S. E., Fraser, H. J., Öberg, K. I., van Dishoeck, E. F., \& Schlemmer, S. 2006, A\&A, 449, 1297

Bisschop, S. E., Jørgensen, J. K., Bourke, T. L., Bottinelli, S., \& van Dishoeck E. F. 2008, A\&A, 488, 959

Bogey, M., Demuynck, C., \& Destombes, J. L. 1986, Chem. Phys. Lett., 125, 383

Bogey, M., Demuynck, C., Destombes, J. L., \& Dubus, H. 1987, J. Mol. Spectrosc., 122, 313

Botschwina, P., Horn, M., Flugge, J., \& Seeger, S. 1993, J. Chem. Soc. Faraday Trans., 89, 2219

Bottinelli, S., Ceccarelli, C., Neri, R., et al. 2004, ApJ, 617, L69

Caratti o Garatti, A., Giannini, T., Nisini, B., \& Lorenzetti, D. 2006, A\&A, 449, 1077

Caselli, P., \& Ceccarelli, C. 2012, A\&ARv, 20, 56

Caselli, P., \& Dore, L. 2005, A\&A, 433, 1145

Cazzoli, G., Cludi, L., Buffa, G., \& Puzzarini, C. 2012, ApJS, 203, 11

Chandra, S., \& Kegel, W. H. 2000, A\&AS, 142, 113

Chen, X., Launhardt, R., \& Henning, T. 2009, ApJ, 691, 1729

Codella, C., Cabrit, S., Gueth, F., et al. 2014, A\&A, 568, L5

Coutens, A., Jørgensen, J. K., van der Wiel, M. H. D., et al. 2016, A\&A, 590, L6

Crapsi, A., Caselli, P., Walmsley, C. M., et al. 2005, ApJ, 619, 379

Crimier, N., Ceccarelli, C., Maret, S., et al. 2010, A\&A, 519, A65

Cuadrado, S., Goicoechea, J. R., Pilleri, P., et al. 2015, A\&A, 575, A82

Di Francesco, J., André, P., \& Myers, P. C. 2004, ApJ, 617, 425

Drozdovskaya, M. N., Walsh, C., Visser, R., Harsono, D., \& van Dishoeck E. F. 2014, MNRAS, 445, 913

Drozdovskaya, M. N., Walsh, C., Visser, R., Harsono, D., \& van Dishoeck E. F. 2015, MNRAS, 451, 3836

Drozdovskaya, M. N., van Dishoeck, E. F., Jørgensen, J. K., et al. 2018, MNRAS, 476, 4949

Dunham, M. M., Stutz, A. M., Allen, L. E., et al. 2014, Protostars and Planets VI (Tucson: University of Arizona Press), 195

Dzib, S. A., Ortiz-León, G. N., Hernández-Gómez, A., et al. 2018, A\&A, 614, A20

Emprechtinger, M., Caselli, P., Volgenau, N. H., Stutzki, J., \& Wiedner, M. C. 2009, A\&A, 493, 89

Endres, C. P., Schlemmer, S., Schilke, P., Stutzki, J., \& Müller, H. S. P. 2016 J. Mol. Spectr., 327, 95
Evans, II, N. J. 1999, ARA\&A, 37, 311

Evans, II, N. J., Rawlings, J. M. C., Shirley, Y. L., \& Mundy, L. G. 2001, ApJ, 557,193

Favre, C., Bergin, E. A., Cleeves, L. I., et al. 2015, ApJ, 802, L23

Favre, C., López-Sepulcre, A., Ceccarelli, C., et al. 2017, A\&A, 608, A82

Fayolle, E. C., Öberg, K. I., Garrod, R. T., van Dishoeck, E. F., \& Bisschop, S. E. 2015, A\&A, 576, A45

Flower, D. R. 1999, MNRAS, 305, 651

Flower, D. R., Pineau Des Forêts, G., \& Walmsley, C. M. 2006, A\&A, 449, 621

Fontani, F., Palau, A., Busquet, G., et al. 2012, MNRAS, 423, 1691

Friesen, R. K., Di Francesco, J., Bourke, T. L., et al. 2014, ApJ, 797, 27

Furuya, K., Aikawa, Y., Tomida, K., et al. 2012, ApJ, 758, 86

Galli, D., Walmsley, M., \& Gonçalves J. 2002, A\&A, 394, 275

Garay, G., Mardones, D., Rodríguez, L. F., Caselli, P., \& Bourke, T. L. 2002, ApJ, 567, 980

Garrod, R. T., \& Herbst, E. 2006, A\&A, 457, 927

Gerin, M., Kaźmierczak, M., Jastrzebska, M., et al. 2011, A\&A, 525, A116

Girart, J. M., Estalella, R., Palau, A., Torrelles, J. M., \& Rao, R. 2014, ApJ, 780, L11

Güsten, R., Nyman, L. Å., Schilke, P., et al. 2006, A\&A, 454, L13

Guzmán, V. V., Pety, J., Goicoechea, J. R., et al. 2015, ApJ, 800, L33

Harsono, D., Jørgensen, J. K., van Dishoeck, E. F., et al. 2014, A\&A, 562, A77

Herbst, E., \& van Dishoeck E. F. 2009, ARA\&A, 47, 427

Hincelin, U., Wakelam, V., Commerçon, B., Hersant, F., \& Guilloteau, S. 2013, ApJ, 775, 44

Hincelin, U., Commerçon, B., Wakelam, V., et al. 2016, ApJ, 822, 12

Ho, P. T. P., Moran, J. M., \& Lo, K. Y. 2004, ApJ, 616, L1

Hogerheijde, M. R., \& van der Tak, F. F. S. 2000, A\&A, 362, 697

Huang, J., Öberg, K. I., Qi, C., et al. 2017, ApJ, 835, 231

Jacobsen, S. K., Jørgensen, J. K., van der Wiel, M. H. D., et al. 2018, A\&A, 612, A72

Jørgensen, J. K., Schöier, F. L., \& van Dishoeck E. F. 2002, A\&A, 389, 908

Jørgensen, J. K., Schöier, F. L., \& van Dishoeck E. F. 2004, A\&A, 416, 603

Jørgensen, J. K., Bourke, T. L., Myers, P. C., et al. 2005a, ApJ, 632, 973

Jørgensen, J. K., Schöier, F. L., \& van Dishoeck E. F. 2005b, A\&A, 437, 501

Jørgensen, J. K., Schöier, F. L., \& van Dishoeck E. F. 2005c, A\&A, 435, 177

Jørgensen, J. K., Bourke, T. L., Nguyen Luong, Q., \& Takakuwa, S. 2011, A\&A 534, A100

Jørgensen, J. K., Visser, R., Sakai, N., et al. 2013, ApJ, 779, L22

Jørgensen, J. K., van der Wiel, M. H. D., Coutens, A., et al. 2016, A\&A, 595, A117

Koumpia, E., van der Tak, F. F. S., Kwon, W., et al. 2016, A\&A, 595, A51

Koumpia, E., Semenov, D. A., van der Tak, F. F. S., Boogert, A. C. A., \& Caux, E. 2017, A\&A, 603, A88

Kristensen, L. E., Klaassen, P. D., Mottram, J. C., Schmalzl, M., \& Hogerheijde, M. R. 2013, A\&A, 549, L6

Li, Z.-Y., Banerjee, R., Pudritz, R. E., et al. 2014, Protostars and Planets VI (Tucson: University of Arizona Press), 173

Lindberg, J. E., Jørgensen, J. K., Brinch, C., et al. 2014a, A\&A, 566, A74

Lindberg, J. E., Jørgensen, J. K., Green, J. D., et al. 2014b, A\&A, 565, A29

Lindberg, J. E., Charnley, S. B., \& Cordiner, M. A. 2016, ApJ, 833, L14

Lindberg, J. E., Charnley, S. B., Jørgensen, J. K., Cordiner, M. A., \& Bjerkeli, P. 2017, ApJ, 835, 3

Liseau, R., Larsson, B., Brandeker, A., et al. 2003, A\&A, 402, L73

Liseau, R., Larsson, B., Lunttila, T., et al. 2015, A\&A, 578, A131

Liszt, H., Sonnentrucker, P., Cordiner, M., \& Gerin, M. 2012, ApJ, 753, L28

Loinard, L., Torres, R. M., Mioduszewski, A. J., \& Rodríguez, L. F. 2008, ApJ, 675, L29

Loinard, L., Zapata, L. A., Rodríguez, L. F., et al. 2013, MNRAS, 430, L10

Lucas, R., \& Liszt, H. S. 2000, A\&A, 358, 1069

Lykke, J. M., Coutens, A., Jørgensen, J. K., et al. 2017, A\&A, 597, A53

Maret, S., Bergin, E. A., \& Lada, C. J. 2006, Nature, 442, 425

Mookerjea, B., Hassel, G. E., Gerin, M., et al. 2012, A\&A, 546, A75

Mookerjea, B., Vastel, C., Hassel, G. E., et al. 2014, A\&A, 566, A61

Müller, H. S. P., Klaus, T., \& Winnewisser, G. 2000, A\&A, 357, L65

Murillo, N. M., \& Lai, S.-P. 2013, ApJ, 764, L15

Murillo, N. M., Lai, S.-P., Bruderer, S., Harsono, D., \& van Dishoeck E. F. 2013, A\&A, 560, A103

Murillo, N. M., Bruderer, S., van Dishoeck, E. F., et al. 2015, A\&A, 579, A114

Murillo, N. M., van Dishoeck, E. F., Tobin, J. J., \& Fedele, D. 2016, A\&A, 592, A56

Nagy, Z., Ossenkopf, V., Van der Tak, F. F. S., et al. 2015, A\&A, 578, A124

Öberg, K. I., Lauck, T., \& Graninger, D. 2014, ApJ, 788, 68

Ortiz-León, G. N., Loinard, L., Kounkel, M. A., et al. 2017, ApJ, 834, 141

Oya, Y., Sakai, N., López-Sepulcre, A., et al. 2016, ApJ, 824, 88

Padovani, M., Walmsley, C. M., Tafalla, M., Galli, D., \& Müller, H. S. P. 2009, A\&A, 505, 1199 
Pagani, L., Daniel, F., \& Dubernet, M.-L. 2009a, A\&A, 494, 719

Pagani, L., Vastel, C., Hugo, E., et al. 2009b, A\&A, 494, 623

Persson, M. V., Harsono, D., Tobin, J. J., et al. 2016, A\&A, 590, A33

Pety, J., Goicoechea, J. R., Hily-Blant, P., Gerin, M., \& Teyssier, D. 2007, A\&A, 464, L41

Pilleri, P., Treviño-Morales, S., Fuente, A., et al. 2013, A\&A, 554, A87

Pineda, J. E., Maury, A. J., Fuller, G. A., et al. 2012, A\&A, 544, L7

Punanova, A., Caselli, P., Pon, A., Belloche, A., \& André, P. 2016, A\&A, 587, A118

Reipurth, B., Clarke, C. J., Boss, A. P., et al. 2014, Protostars and Planets VI (Tucson: University of Arizona Press), 267

Sakai, N., \& Yamamoto, S. 2013, Chem. Rev., 113, 8981

Sakai, N., Sakai, T., Hirota, T., \& Yamamoto, S. 2010, ApJ, 722, 1633

Sakai, N., Oya, Y., Sakai, T., et al. 2014a, ApJ, 791, L38

Sakai, N., Sakai, T., Hirota, T., et al. 2014b, Nature, 507, 78

Sakai, N., Oya, Y., López-Sepulcre, A., et al. 2016, ApJ, 820, L34

Salinas, V. N., Hogerheijde, M. R., Mathews, G. S., et al. 2017, A\&A, 606, A125

Santangelo, G., Murillo, N. M., Nisini, B., et al. 2015, A\&A, 581, A91

Sastry, K. V. L. N., Helminger, P., Charo, A., Herbst, E., \& De Lucia F. C. 1981, ApJ, 251, L119

Schöier, F. L., Jørgensen, J. K., van Dishoeck, E. F., \& Blake, G. A. 2002, A\&A, 390, 1001

Schöier, F. L., van der Tak, F. F. S., van Dishoeck, E. F., \& Black, J. H. 2005, A\&A, 432, 369

Shirley, Y. L. 2015, PASP, 127, 299

Spezzano, S., Tamassia, F., Thorwirth, S., et al. 2012, ApJS, 200, 1

Spezzano, S., Bizzocchi, L., Caselli, P., Harju, J., \& Brünken, S. 2016a, A\&A 592, L11
Spezzano, S., Gupta, H., Brünken, S., et al. 2016b, A\&A, 586, A110 Stark, R., Sandell, G., Beck, S. C., et al. 2004, ApJ, 608, 341

Tassis, K., Willacy, K., Yorke, H. W., \& Turner, N. J. 2012, ApJ, 753, 29

Tobin, J. J., Hartmann, L., Chiang, H.-F., et al. 2012, Nature, 492, 83

Tobin, J. J., Bergin, E. A., Hartmann, L., et al. 2013, ApJ, 765, 18

van der Tak, F. F. S., Black, J. H., Schöier, F. L., Jansen, D. J., \& van Dishoeck E. F. 2007, A\&A, 468, 627

van der Wiel, M. H. D., van der Tak, F. F. S., Ossenkopf, V., et al. 2009, A\&A, 498, 161

van Dishoeck, E. F., Jansen, D. J., \& Phillips, T. G. 1993, A\&A, 279, 541

van Dishoeck, E. F., Blake, G. A., Jansen, D. J., \& Groesbeck, T. D. 1995, ApJ, 447, 760

van't Hoff, M. L. R., Walsh, C., Kama, M., Facchini, S., \& van Dishoeck E. F. 2017, A\&A, 599, A101

Vassilev, V., Meledin, D., Lapkin, I., et al. 2008, A\&A, 490, 1157

Visser, R., van Dishoeck, E. F., Doty, S. D., \& Dullemond, C. P. 2009, A\&A, 495, 881

Visser, R., Doty, S. D., \& van Dishoeck E. F. 2011, A\&A, 534, A132

Walmsley, C. M., Flower, D. R., \& Pineau des Forêts, G. 2004, A\&A, 418, 1035

Willacy, K., Alexander, C., Ali-Dib, M., et al. 2015, Space Sci. Rev., 197, 151

Wootten, A., Andre, P., Despois, D., \& Sargent, A. 1994, in Clouds, Cores, and Low Mass Stars, eds. D. P. Clemens, \& R. Barvainis, ASP Conf. Ser., 65, 294

Yeh, S. C. C., Hirano, N., Bourke, T. L., et al. 2008, ApJ, 675, 454

Yen, H.-W., Koch, P. M., Takakuwa, S., et al. 2017, ApJ, 834, 178

Yoneda, H., Tsukamoto, Y., Furuya, K., \& Aikawa, Y. 2016, ApJ, 833, 105

Zapata, L. A., Loinard, L., Rodríguez, L. F., et al. 2013, ApJ, 764, L14 


\section{Appendix A: Interferometric observations}

Table A.1. Interferometric observations.

\begin{tabular}{|c|c|c|c|c|c|c|}
\hline Dataset & $\begin{array}{c}\text { Frequency } \\
(\mathrm{GHz})\end{array}$ & $\begin{array}{l}\text { UV-baseline range } \\
\qquad(\mathrm{k} \lambda)\end{array}$ & $\begin{array}{c}\text { Beam } \\
\left({ }^{\prime \prime}\right)\end{array}$ & $\begin{array}{c}\text { Largest scale } \\
\left({ }^{\prime \prime}\right)\end{array}$ & $\begin{array}{c}\text { Field of view } \\
\left({ }^{\prime \prime}\right)\end{array}$ & $\begin{array}{c}\text { Noise } \\
\left(\mathrm{mJy}^{-1} \text { beam }^{-1}\right)\end{array}$ \\
\hline \multicolumn{7}{|c|}{ IRAS $16293-2422$} \\
\hline Combined $12 \mathrm{~m}+\mathrm{ACA}$ & $329.1-362.8$ & $10 \sim 352$ & $0.5 \times 0.5$ & 13 & 19.5 & $7-10$ \\
\hline $\mathrm{ACA}$ & $329.1-362.8$ & $10 \sim 60$ & $5.25 \times 2.36$ & 13 & 60 & 440 \\
\hline SMA Compact & $215.6-227.6$ & $6 \sim 91$ & $5.5 \times 3.2$ & 20 & 64 & 240 \\
\hline \multicolumn{7}{|c|}{ VLA $1623-2217$} \\
\hline Cycle $012 \mathrm{~m}$ & $216.4-232.2$ & $25 \sim 310$ & $0.85 \times 0.56$ & 2.5 & 28 & $4-8$ \\
\hline Cycle 2 Band $612 \mathrm{~m} \mathrm{C} 35-5$ & $215.5-221.4$ & $18 \sim 791$ & $0.45 \times 0.25$ & 1.9 & 28 & $7-9$ \\
\hline Cycle 2 Band 612 m C34-1 & $215.5-221.4$ & $11 \sim 253$ & $1.6 \times 0.88$ & 5.3 & 28 & 20 \\
\hline Cycle 2 Band $712 \mathrm{~m}$ & $359.0-372.7$ & $18 \sim 420$ & $0.87 \times 0.54$ & 3.1 & 17.2 & $26-95$ \\
\hline
\end{tabular}

Table A.1 lists details of the interferometric observations used in this work for both systems, IRAS 16293 and VLA 1623. For VLA 1623, the Cycle 2 Band $612 \mathrm{~m}$ array observations have both long (C35-5) and short (C34-1) baselines. The short baselines were observed to bridge the baseline ranges between the ACA and $12 \mathrm{~m}$ observations. The $\mathrm{c}-\mathrm{C}_{3} \mathrm{H}_{2}$ observations toward VLA 1623 presented in this work were detected with the $12 \mathrm{~m}$ array short baselines but not the long baselines, suggesting that the emission arises from regions larger than $\sim 0.5^{\prime \prime}$.

\section{Appendix B: Additional $\mathrm{C}-\mathrm{C}_{3} \mathrm{H}_{2}$ and $\mathrm{C}_{2} \mathrm{H}$ spectra}

The spectra for all transitions of $\mathrm{c}-\mathrm{C}_{3} \mathrm{H}_{2}$ and $\mathrm{C}_{2} \mathrm{H}$ toward the south, center, and north positions of IRAS 16293 are shown in Fig. B.1. The systemic velocity of IRAS 16293 A and B are marked on the spectra with dashed lines. The anti-correlation of the two molecules is evident from the spectra, as well as a slight velocity shift in $\mathrm{C}_{2} \mathrm{H}$ between the center and north positions. 

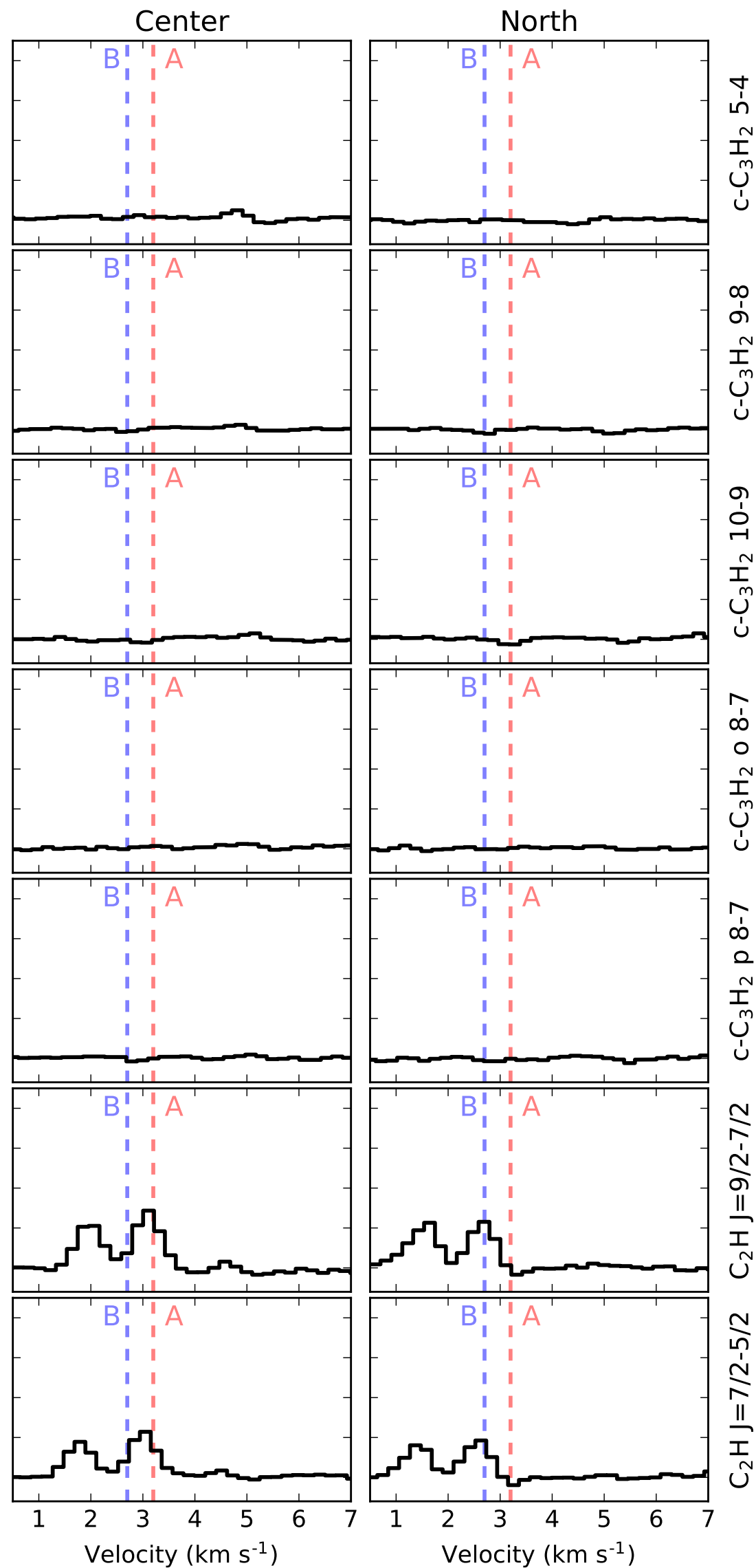

Fig. B.1. IRAS 16293-2422 ALMA spectra for all transitions of $\mathrm{c}-\mathrm{C}_{3} \mathrm{H}_{2}$ and $\mathrm{C}_{2} \mathrm{H}$ for each of the south, center, and north positions shown in Fig. 3 . The systemic velocities of sources A and B are marked with the vertical dashed lines. The anti-correlation of $c-\mathrm{C}_{3} \mathrm{H}_{2}$ and $\mathrm{C}_{2} \mathrm{H}$ is also evident in the spectra shown here. 


\section{Appendix C: Peak intensities and line ratios}

Table C.1. $\mathrm{c}-\mathrm{C}_{3} \mathrm{H}_{2}$ and $\mathrm{C}_{2} \mathrm{H}$ peak intensities.

\begin{tabular}{|c|c|c|c|c|c|c|c|c|c|}
\hline \multirow{2}{*}{$\begin{array}{l}\text { Molecules } \\
\text { Transition }\end{array}$} & \multicolumn{5}{|c|}{$\mathrm{c}-\mathrm{C}_{3} \mathrm{H}_{2}$} & \multicolumn{4}{|c|}{$\mathrm{C}_{2} \mathrm{H}$} \\
\hline & $5-4$ & $9-8$ & $10-9$ & $8_{2,6}-7_{3,5}$ & $8_{3,6}-7_{2.5}$ & $J=9 / 2-7 / 2 F=5-4$ & $J=9 / 2-7 / 2 F=4-3$ & $J=7 / 2-5 / 2 F=4-3$ & $J=7 / 2-5 / 2 F=3-2$ \\
\hline \multicolumn{10}{|c|}{ IRAS $16293-2422\left(\mathrm{rms}=10 \mathrm{mJy}\right.$ beam $\left.^{-1}\right)$} \\
\hline South $^{a}$ & 240 & 410 & 350 & 90 & 200 & 30 & 30 & 30 & 30 \\
\hline Center $a$ & 30 & 30 & 30 & 30 & 30 & 170 & 140 & 140 & 110 \\
\hline North $^{a}$ & 30 & 30 & 30 & 30 & 30 & 150 & 130 & 110 & 110 \\
\hline \multicolumn{10}{|c|}{ VLA $1623-2417-\operatorname{APEX}(\mathrm{rms}=80 \mathrm{mK})$} \\
\hline A & $\ldots$ & $\ldots$ & $\ldots$ & $\ldots$ & $\ldots$ & 960 & 616 & 680 & 342 \\
\hline
\end{tabular}

Notes. ${ }^{(a)}$ For the positions with nondetections, three times the rms noise is used for the calculations.

Table C.2. $\mathrm{N}_{2} \mathrm{H}^{+}$and $\mathrm{N}_{2} \mathrm{D}^{+}$parameters for VLA $1623-2417$.

\begin{tabular}{|c|c|c|c|c|}
\hline \multirow[b]{2}{*}{ Parameter } & \multicolumn{4}{|c|}{ Observed } \\
\hline & $\mathrm{N}_{2} \mathrm{H}^{+} 1-0^{a}$ & $\mathrm{~N}_{2} \mathrm{H}^{+} 6-5^{b}$ & $\mathrm{~N}_{2} \mathrm{D}^{+} 1-0^{a}$ & $\mathrm{~N}_{2} \mathrm{D}^{+} 2-1^{a}$ \\
\hline$T_{\mathrm{mb}}(\mathrm{K})$ & $3.3 \pm 0.1$ & $0.2 \pm 0.1$ & $0.57 \pm 0.1$ & $1.5 \pm 0.2$ \\
\hline$\Delta v_{\text {single dish }}\left(\mathrm{km} \mathrm{s}^{-1}\right)$ & $0.56 \pm 0.005$ & $0.7 \pm 0.2$ & $0.55 \pm 0.032$ & $0.55 \pm 0.009$ \\
\hline$\eta_{\mathrm{mb}}$ & 0.95 & 0.63 & 0.95 & 0.94 \\
\hline$\Omega_{\text {beam }}\left({ }^{\prime \prime}\right)$ & 26.5 & 36 & 32.1 & 16.3 \\
\hline Ratio & \multicolumn{2}{|c|}{$0.06 \pm 0.03$} & \multicolumn{2}{|c|}{$2.6 \pm 0.6$} \\
\hline Column density $\left(\mathrm{cm}^{-2}\right)$ & \multicolumn{2}{|c|}{$1.3 \times 10^{13}$} & \multicolumn{2}{|c|}{$1.8 \times 10^{12}$} \\
\hline $\mathrm{H}_{2}$ density $\left(\mathrm{cm}^{-3}\right)$ & \multicolumn{2}{|c|}{$5 \times 10^{7}-7 \times 10^{9}$} & \multicolumn{2}{|c|}{$5 \times 10^{7}-7 \times 10^{9}$} \\
\hline$T_{\mathrm{ex}}(\mathrm{K})$ & \multicolumn{2}{|c|}{$11-12$} & \multicolumn{2}{|c|}{$11-12$} \\
\hline$\Omega_{\text {source }}=\Omega_{\text {beam }}$ & \multicolumn{2}{|c|}{$\mathrm{N}_{2} \mathrm{H}^{+} 4-3$} & \multicolumn{2}{|c|}{$\mathrm{N}_{2} \mathrm{D}^{+} 3-2$} \\
\hline$I_{\nu, \text { predicted }}\left(\mathrm{mJy} \mathrm{beam}^{-1}\right)$ & \multicolumn{2}{|c|}{99} & \multicolumn{2}{|c|}{22} \\
\hline$\Omega_{\text {beam,obs }}\left({ }^{\prime \prime}\right)$ & \multicolumn{2}{|c|}{0.85} & \multicolumn{2}{|c|}{0.76} \\
\hline$\sigma_{\text {obs }}\left(\mathrm{mJy} \mathrm{beam}^{-1}\right)$ & \multicolumn{2}{|c|}{94.9} & \multicolumn{2}{|c|}{8.58} \\
\hline$S / N$ & \multicolumn{2}{|c|}{1} & \multicolumn{2}{|c|}{2.6} \\
\hline$\Omega_{\text {source }}=1^{\prime \prime}$ & \multicolumn{2}{|c|}{$\mathrm{N}_{2} \mathrm{H}^{+} 4-3$} & \multicolumn{2}{|c|}{$\mathrm{N}_{2} \mathrm{D}^{+} 3-2$} \\
\hline$I_{v, \text { predicted }}\left(\mathrm{mJy}\right.$ beam $\left.^{-1}\right)$ & \multicolumn{2}{|c|}{2631} & \multicolumn{2}{|c|}{364} \\
\hline$\Omega_{\text {beam,obs }}\left({ }^{\prime \prime}\right)$ & \multicolumn{2}{|c|}{0.85} & \multicolumn{2}{|c|}{0.76} \\
\hline$\sigma_{\text {obs }}\left(\mathrm{mJy} \mathrm{beam}^{-1}\right)$ & \multicolumn{2}{|c|}{94.9} & \multicolumn{2}{|c|}{8.58} \\
\hline$S / N$ & \multicolumn{2}{|c|}{27} & \multicolumn{2}{|c|}{42} \\
\hline
\end{tabular}

Notes. ${ }^{(a)}$ IRAM 30 m observations from Punanova et al. (2016). ${ }^{(b)}$ Herschel observations from Favre et al. (2017).

The observed peak intensities of $\mathrm{C}_{2} \mathrm{H}$ and $\mathrm{c}-\mathrm{C}_{3} \mathrm{H}_{2}$ are listed in this appendix (Table C.1). The peak intensities are used in Sects. 4.2 and 4.3. In addition, the detailed calculation of expected peak intensities for $\mathrm{N}_{2} \mathrm{H}^{+}$and $\mathrm{N}_{2} \mathrm{D}^{+}$are also summarized here (Table C.2).

Single-dish observations of low- $J$ transitions of $\mathrm{CN}, \mathrm{HCN}$, and HNC show strong detections relative to other embedded systems (Jørgensen et al. 2004). Our recent APEX observations also detected NO toward VLA 1623. However, single-dish observations of $\mathrm{NH}_{3}$ (Wootten et al. 1994; Liseau et al. 2003) and $\mathrm{N}_{2} \mathrm{H}^{+}$(Liseau et al. 2015; Punanova et al. 2016) indicate that these molecules have very low abundances at the position of VLA 1623. $\mathrm{N}_{2} \mathrm{D}^{+}$also exhibits the same behavior (Punanova et al. 2016). Furthermore, Herschel observations of high- $J \mathrm{~N}_{2} \mathrm{H}^{+}$ toward VLA 1623 (Liseau et al. 2015; Favre et al. 2017) show that the molecule is detected up to the $J=6-5$ transition peaking at $\sim 0.1 \mathrm{~K} \mathrm{~km} \mathrm{~s}^{-1}$ but the emission is extended. The observed parameters of $\mathrm{N}_{2} \mathrm{H}^{+}$and $\mathrm{N}_{2} \mathrm{D}^{+}$from Punanova et al. (2016) and Favre et al. (2017) are listed in Table C.2.

Using the observed transitions of $\mathrm{N}_{2} \mathrm{H}^{+}$and $\mathrm{N}_{2} \mathrm{D}^{+}$, we derive density and excitation temperature with the method described in Sect. 4.1. The different beam sizes of the observations require a beam dilution correction factor that is given by $T_{\mathrm{mb}}^{\prime}=T_{\mathrm{mb}, \mathrm{obs}} \frac{\Omega_{\text {beam }}}{\Omega_{\text {source }}}$, where $T_{\mathrm{mb}}^{\prime}$ and $T_{\mathrm{mb}, \mathrm{obs}}$ are the corrected and observed main beam temperature, respectively, $\Omega_{\text {beam }}$ is the solid angle of the single-dish beam and $\Omega_{\text {source }}$ is the solid angle subtended by the source. We assume the emission is concentrated in the region of the smaller beam, which would be of $26.5^{\prime \prime}$ for $\mathrm{N}_{2} \mathrm{H}^{+}$and $16.3^{\prime \prime}$ for $\mathrm{N}_{2} \mathrm{D}^{+}$.

To compare with our ALMA observations, the expected peak for $\mathrm{N}_{2} \mathrm{H}^{+} 4-3$ and $\mathrm{N}_{2} \mathrm{D}^{+} 3-2$ is derived from the observations of Punanova et al. (2016) and Favre et al. (2017). The $\mathrm{N}_{2} \mathrm{H}^{+}$molecular data file without hyperfine structure is used to calculate the kinetic temperature of both molecules. The collisional rate coefficients for $\mathrm{N}_{2} \mathrm{H}^{+}$are taken to be the same as $\mathrm{HCO}^{+}$(Botschwina et al. 1993) and extrapolated (Schöier et al. 2005). Since LAMDA does not have a molecular data file for $\mathrm{N}_{2} \mathrm{D}^{+}$, the data file for $\mathrm{N}_{2} \mathrm{H}^{+}$is used, selecting the corresponding transition rather than frequency. For the predicted peak emissions for $\mathrm{N}_{2} \mathrm{H}^{+} 4-3$ and $\mathrm{N}_{2} \mathrm{D}^{+} 3-2$, two cases are examined: (i) the observed emission is evenly distributed in the singledish beam (i.e., beam-filling factor $=1$ ) and (ii) the emission is 
concentrated in a $1^{\prime \prime}$ region (i.e., beam filling factor $<1$ ). The second case introduces a beam dilution correction. The results of the calculation are listed in Table C.2.

The kinetic temperature and number density obtained in our calculations $\left(\sim 11 \mathrm{~K}, \sim 10^{7-9} \mathrm{~cm}^{-3}\right)$ are slightly higher than those previously reported $\left(7.7 \mathrm{~K}, 10^{6} \mathrm{~cm}^{-3}\right.$; Punanova et al. 2016). The column densities, however, are similar to those reported in Punanova et al. (2016). For $\mathrm{N}_{2} \mathrm{H}^{+}$, our results are also consistent with those reported in Liseau et al. (2015).

Since only one transition of $\mathrm{N}_{2} \mathrm{D}^{+}$toward IRAS 16293 is presented here, the physical parameters of other molecules are used to derive its column density. Using the physical parameters obtained from the $\mathrm{DCO}^{+} 5-4 / 3-2$ ratio (Sect. 4.1 and Table 4), we calculated a column density of $1.5-2 \times 10^{13} \mathrm{~cm}^{-2}$ for $\mathrm{N}_{2} \mathrm{D}^{+}$. If instead we used the physical parameters obtained from the
$\mathrm{N}_{2} \mathrm{D}^{+}$observations toward VLA 1623, we find a column density of $4-5 \times 10^{13} \mathrm{~cm}^{-2}$ for $\mathrm{N}_{2} \mathrm{D}^{+} 3-2$ toward IRAS 16293 . For both sets of parameters, the column density is higher by one order of magnitude in comparison to the $\mathrm{N}_{2} \mathrm{D}^{+}$toward VLA 1623.

\section{Appendix D: PILS full spectra}

The PILS survey spectra (Jørgensen et al. 2016) is reproduced here for the south $\mathrm{c}-\mathrm{C}_{3} \mathrm{H}_{2}$ peak position and at one beam away from the position of IRAS 16293 B . Figures D.1-D.3 present the full spectra for both positions. At the south $\mathrm{c}-\mathrm{C}_{3} \mathrm{H}_{2}$ peak position, the spectra is multiplied by a factor of 10 to bring out the features. Few molecular lines are detected at this position, apart from common molecules like $\mathrm{HCO}^{+}$and $\mathrm{CO}$, only $\mathrm{c}-\mathrm{C}_{3} \mathrm{H}_{2}$ and $\mathrm{H}_{2} \mathrm{CS}$ are detected. 
N. M. Murillo et al.: Tracing the physico-chemical structure of IRAS 16293-2422 and VLA 1623-2417
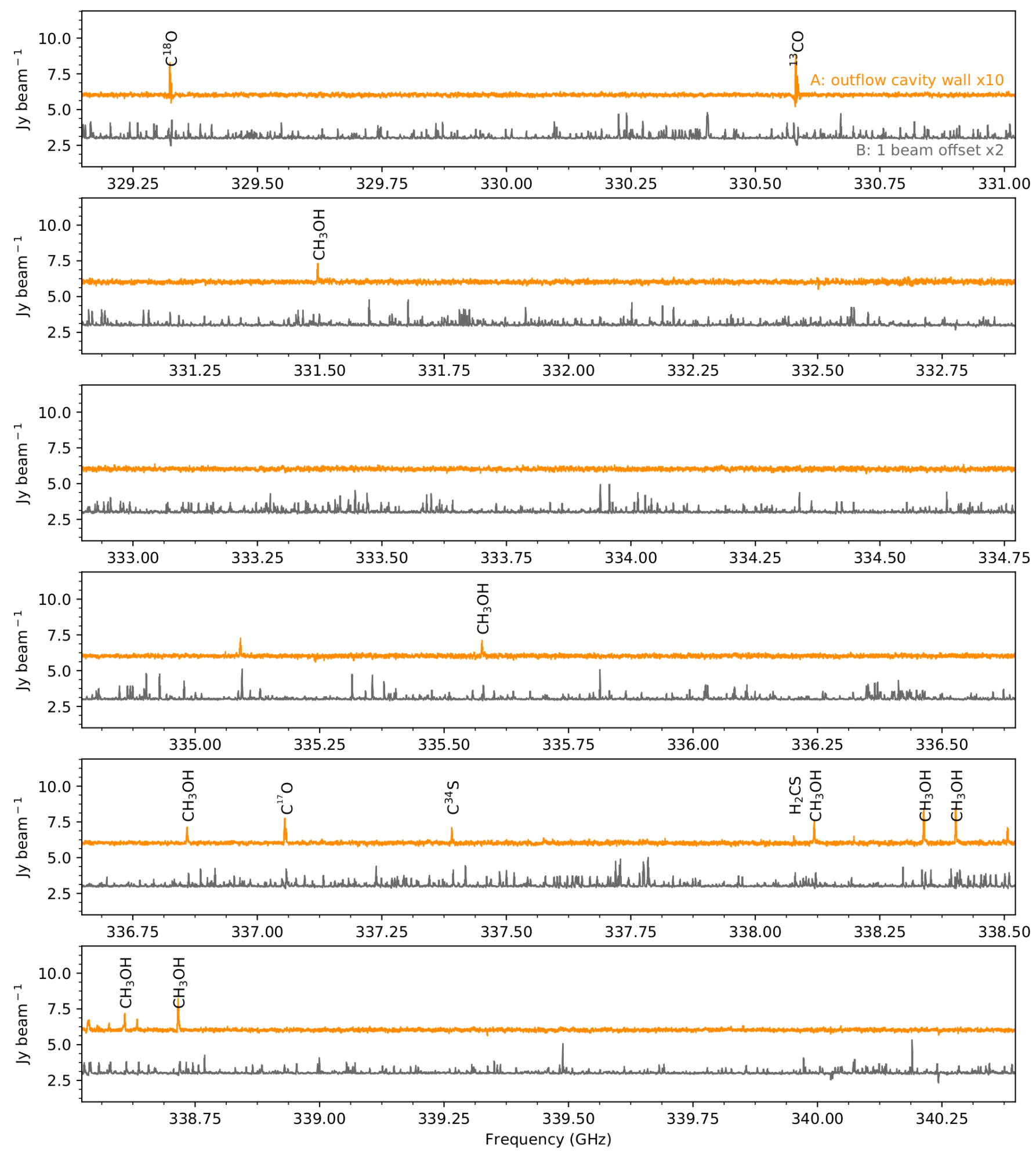

Fig. D.1. Full spectra obtained in the PILS survey for IRAS 16293-2422. Here, the frequency range 329.15-340.4 GHz is shown. The rest of the spectra is shown in Figs. D.2 and D.3. The spectra for the IRAS 16293-2422 B is one beam away from the source position $\left(\alpha_{J 2000}=16: 32: 22.581\right.$, $\delta_{J 2000}=-24: 28: 32.80$ ), we note that it is multiplied by a factor of 2. The spectra for IRAS 16293-2422 A's outflow cavity wall is centered at the observed peak of $\mathrm{c}-\mathrm{C}_{3} \mathrm{H}_{2}\left(\alpha_{J 2000}=16: 32: 22.867, \delta_{J 2000}=-24: 28: 39.60\right)$, we note that it is multiplied by a factor of 10 and shows very little emission other than $\mathrm{c}-\mathrm{C}_{3} \mathrm{H}_{2}, \mathrm{CH}_{3} \mathrm{OH}, \mathrm{CS}, \mathrm{H}_{2} \mathrm{CS}, \mathrm{HCN}, \mathrm{H}_{2} \mathrm{CO}$, and $\mathrm{HCO}^{+}$. 
A\&A 617, A120 (2018)
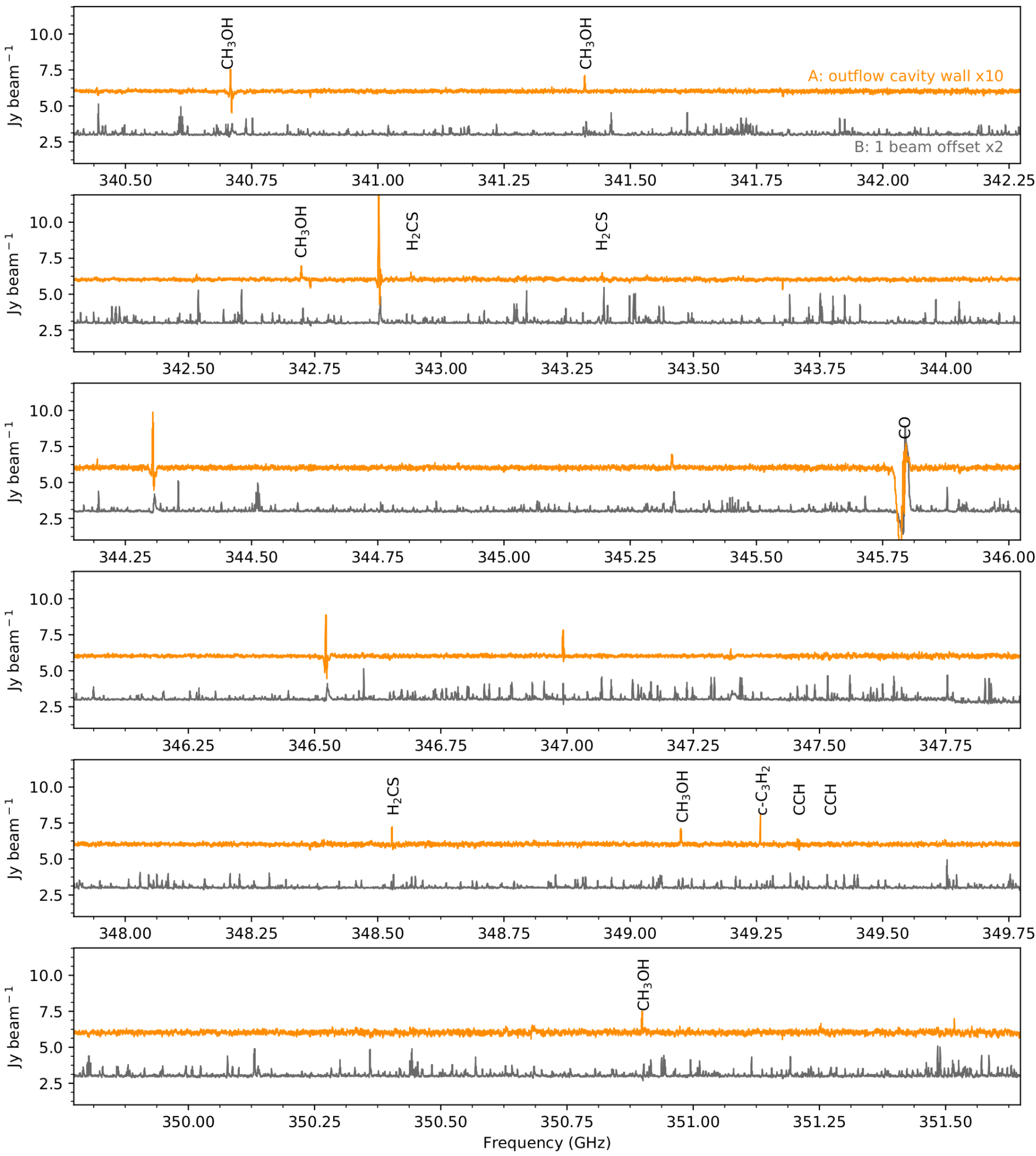

Fig. D.2. Same as in Fig. D.1 but for the frequency range 340.4-351.65 GHz. $\mathrm{C}_{2} \mathrm{H}$ is marked for reference. 
N. M. Murillo et al.: Tracing the physico-chemical structure of IRAS 16293-2422 and VLA 1623-2417
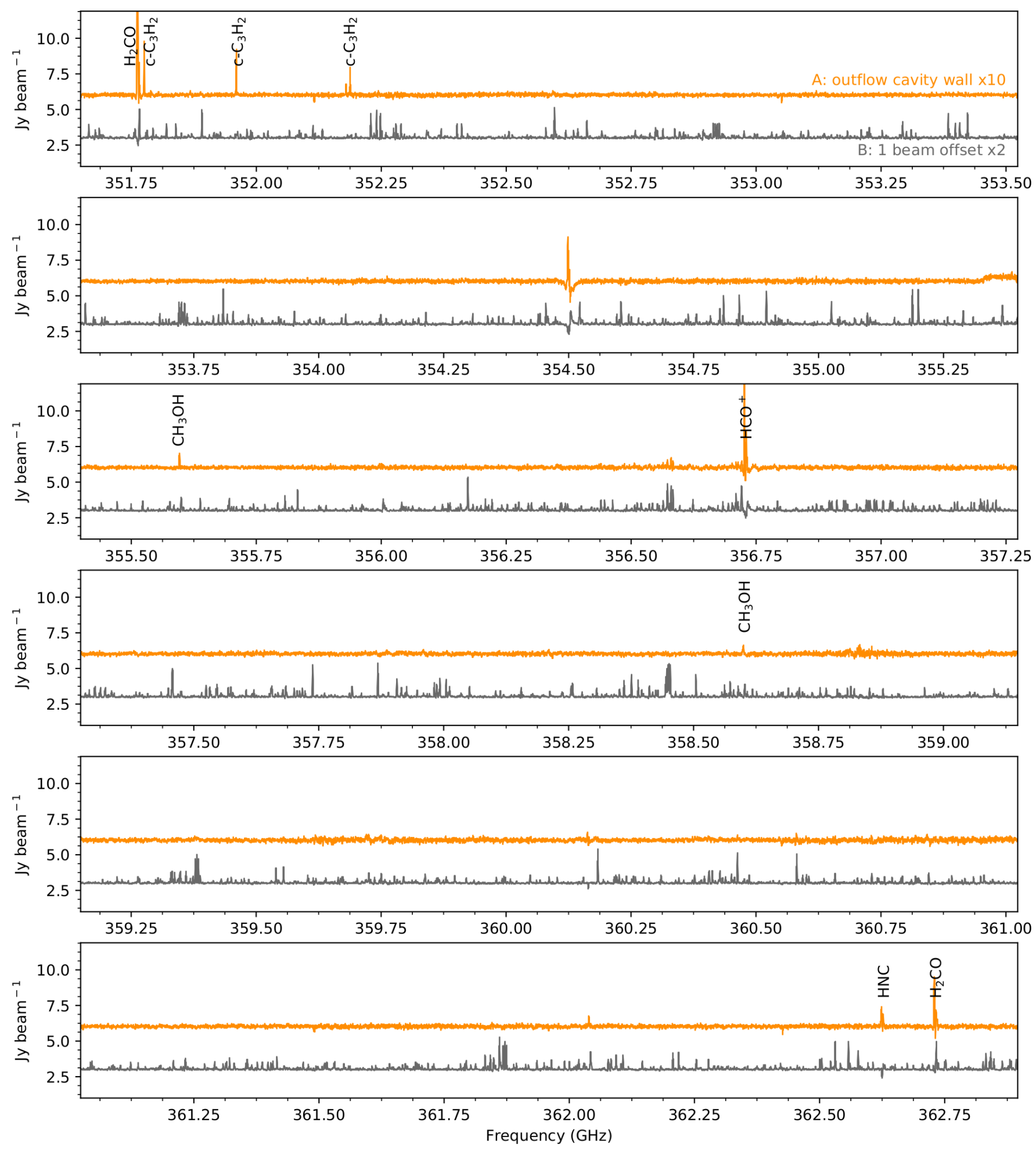

Fig. D.3. Same as in Fig. D.1 but for the frequency range 351.65-362.9 GHz. 